\title{
Global Boundedness of the Gradient for a Class of Schrödinger Equations
}

\author{
Sibei Yang
}

\begin{abstract}
In this paper, via applying the method developed by A. Cianchi and V. Maz'ya, the author obtains the global boundedness of the gradient for solutions to Dirichlet and Neumann problems of a class of Schrödinger equations under the minimal assumptions for integrability on the data and regularity on the boundary of the domain. Moreover, the case of arbitrary bounded semi-convex domains is also considered.
\end{abstract}

\section{Introduction}

It is well known that the global regularity of solutions is a classical topic in the theory of elliptic PDEs. In particular, the study for the global boundedness of the gradient, equivalently, the Lipschitz continuity, of the solutions to some elliptic boundary value problems has attracted great interests in recent years (see, for example, $[1,3,5,6,7,8$, 10, 17, 18]). This topic can be traced back to the work in $[19,26,27]$.

Via a relative isoperimetric inequality (see, for example, $[6,(5.1)]$ or $(2.1)$ below), the Hardy-Littlewood inequality for rearrangements (see, for example, (1.11) below) and a differential inequality involving integrals over the level sets of Sobolev functions established by Maz'ya [19], Cianchi and Maz'ya [6, 8] studied the global boundedness of the gradient for a class of quasi-linear elliptic equations or systems under the weakest integrability conditions on the prescribed date and the minimal regularity assumptions on the domains.

In this paper, motivated by the work in $[6,8,17,18]$, via applying the method developed by Cianchi and Maz'ya [6, 8] and some estimates obtained by Shen [24, 25], we study the global boundedness of the gradient for a class of Schrödinger equations with the Dirichlet or Neumann boundary condition under the minimal assumptions for the integrability on the prescribed dates and the regularity on the domains. Furthermore, the case of arbitrary bounded semi-convex domains is also included.

To state Schrödinger equations considered in this paper, we first recall the definition of the reverse Hölder class (see, for example, $[12,24]$ ). Recall that a non-negative function $w$ on $\mathbb{R}^{n}$ is said to belong to the reverse Hölder class $R H_{q}\left(\mathbb{R}^{n}\right)$ with $q \in(1, \infty]$, denoted by $w \in R H_{q}\left(\mathbb{R}^{n}\right)$ if, when $q \in(1, \infty), w \in L_{\mathrm{loc}}^{q}\left(\mathbb{R}^{n}\right)$ and

$$
\sup _{B \subset \mathbb{R}^{n}}\left\{\frac{1}{|B|} \int_{B}[w(x)]^{q} d x\right\}^{1 / q}\left\{\frac{1}{|B|} \int_{B} w(x) d x\right\}^{-1}<\infty
$$

2010 Mathematics Subject Classification. Primary 35J10; Secondary 35B65, 35J25, 35D30, 35 B45.

Key words and phrases. Schrödinger equation, Dirichlet problem, Neumann problem, boundedness of the gradient, isoperimetric inequality, semi-convex domain.

Sibei Yang is supported by the National Natural Science Foundation of China (Grant Nos. 11401276 and 11571289). 
or, when $q=\infty, w \in L_{\text {loc }}^{\infty}\left(\mathbb{R}^{n}\right)$ and

$$
\sup _{B \subset \mathbb{R}^{n}}\left\{\operatorname{esssup}_{x \in B} w(x)\right\}\left\{\frac{1}{|B|} \int_{B} w(x) d x\right\}^{-1}<\infty,
$$

where the suprema are taken over all balls $B \subset \mathbb{R}^{n}$. A typical example of the reverse Hölder class is a non-negative polynomial on $\mathbb{R}^{n}$, which turns out to be in $R H_{\infty}\left(\mathbb{R}^{n}\right.$ ) (see, for example, [24]). It is known from [12] that $R H_{q}\left(\mathbb{R}^{n}\right)$ has the property of self-improvement. Namely, if $V \in R H_{q}\left(\mathbb{R}^{n}\right)$ with some $q \in(1, \infty)$, then there exists $\epsilon \in(0, \infty)$ such that $V \in R H_{q+\epsilon}\left(\mathbb{R}^{n}\right)$. Thus, for any $V \in R H_{q}\left(\mathbb{R}^{n}\right)$ with $q \in(1, \infty]$, the critical index $q_{+}$for $V$ is defined as follows:

$$
q_{+}:=\sup \left\{q \in(1, \infty]: V \in R H_{q}\left(\mathbb{R}^{n}\right)\right\} .
$$

Let $n \geq 3$ and $\Omega$ be a bounded domain in $\mathbb{R}^{n}$. Denote by $W^{1,2}(\Omega)$ and $W_{0}^{1,2}(\Omega)$ be the classical Sobolev space on $\Omega$ and the closure of $C_{c}^{\infty}(\Omega)$ in $W^{1,2}(\Omega)$, respectively, where $C_{c}^{\infty}(\Omega)$ denotes the set of all $C^{\infty}$ functions on $\mathbb{R}^{n}$ with compact support contained in $\Omega$. Assume that $0 \leq V \in R H_{q}\left(\mathbb{R}^{n}\right)$ for some $q \in[n, \infty]$ and $V \not \equiv 0$ on $\Omega$. Let $f \in L^{2}(\Omega)$. Then $u \in W_{0}^{1,2}(\Omega)$ is said to be a weak solution to the Dirichlet boundary problem

$$
\begin{cases}-\Delta u+V u=f & \text { in } \Omega \\ u=0 & \text { on } \partial \Omega\end{cases}
$$

if for any $v \in W_{0}^{1,2}(\Omega)$,

$$
\int_{\Omega} \nabla u(x) \cdot \nabla v(x) d x+\int_{\Omega} V(x) u(x) v(x) d x=\int_{\Omega} f(x) v(x) d x .
$$

Assume further that $\Omega$ is a bounded Lipschitz domain in $\mathbb{R}^{n}$. Then $u \in W^{1,2}(\Omega)$ is said to be a weak solution of the Neumann boundary problem

$$
\begin{cases}-\Delta u+V u=f & \text { in } \Omega, \\ \frac{\partial u}{\partial \nu}=0 & \text { on } \partial \Omega,\end{cases}
$$

where and in what follows, $\nu:=\left(\nu_{1}, \ldots, \nu_{n}\right)$ denotes the outward unit normal to $\partial \Omega$, if for any $v \in W^{1,2}(\Omega)$, (1.3) holds true.

Remark 1.1. Let $f \in L^{2}(\Omega), 0 \leq V \in R H_{n}\left(\mathbb{R}^{n}\right)$ and $V \not \equiv 0$ on $\Omega$. Assume that $u$ is a weak solution of (1.2) or (1.4).

(i) By (1.3), we conclude that the weak solutions of the Dirichlet problem (1.2) and the Neumann problem (1.4) are unique.

(ii) By Sobolev's inequality and the method of difference quotient, similar to the classical regularity for second order elliptic equation (see, for example, [13, Theorem 8.8]), we know that, for any $\Omega^{\prime} \subset \Omega$ with $\overline{\Omega^{\prime}} \subset \Omega, u \in W^{2,2}\left(\Omega^{\prime}\right)$ and there exists a positive constant $C$, depending on $n, V, \Omega$ and $\Omega^{\prime}$, such that

$$
\|u\|_{W^{2,2}\left(\Omega^{\prime}\right)} \leq C\left(\|u\|_{W^{1,2}(\Omega)}+\|f\|_{L^{2}(\Omega)}\right) .
$$

(iii) Assume further that $\partial \Omega \in C^{2}$. Then similar to the global regularity for second order elliptic equation (see, for example, [13, Theorem 8.12]), we conclude that $u \in W^{2,2}(\Omega)$. 
Then the main results of this paper are as follows.

Theorem 1.2. Let $n \geq 3$ and $\Omega$ be a bounded domain in $\mathbb{R}^{n}$ with $\partial \Omega \in W^{2} L^{n-1,1}$. Assume that $0 \leq V \in R H_{n}\left(\mathbb{R}^{n}\right)$ and $V \not \equiv 0$ on $\Omega, f \in L^{n, 1}(\Omega)$ and $u$ is the unique weak solution to the Dirichlet problem (1.2). Then there exists a positive constant $C$, depending on $n$ and $\Omega$, such that

$$
\|\nabla u\|_{L^{\infty}(\Omega)} \leq C\|f\|_{L^{n, 1}(\Omega)} .
$$

In particular, $u$ is Lipschitz continuous in $\Omega$.

An argument similar to Theorem 1.2 yields that the conclusion of Theorem 1.2 also holds true in the case of bounded semi-convex domains (see Definition 1.7 below for the definition of semi-convex domains).

Theorem 1.3. The same conclusion as in Theorem 1.2 holds true if $\Omega$ is a bounded semi-convex domain in $\mathbb{R}^{n}$ with $n \geq 3$.

Theorem 1.4. Let $n \geq 3$ and $\Omega$ be a bounded domain in $\mathbb{R}^{n}$ with $\partial \Omega \in W^{2} L^{n-1,1}$. Assume that $0 \leq V \in R H_{n}\left(\mathbb{R}^{n}\right)$ and $V \not \equiv 0$ on $\Omega, f \in L^{n, 1}(\Omega)$ and $u$ is the unique weak solution to the Neumann problem (1.4). Then there exists a positive constant $C$, depending on $n$ and $\Omega$, such that

$$
\|\nabla u\|_{L^{\infty}(\Omega)} \leq C\|f\|_{L^{n, 1}(\Omega)} .
$$

In particular, $u$ is Lipschitz continuous in $\Omega$.

Similarly, the conclusion of Theorem 1.4 also holds true in the case of bounded semiconvex domains.

Theorem 1.5. The same conclusion as in Theorem 1.4 holds true if $\Omega$ is a bounded semi-convex domain in $\mathbb{R}^{n}$ with $n \geq 3$.

We prove Theorems $1.2-1.5$ by using the method developed by A. Cianchi and V. Maz'ya $[6,8]$. More precisely, via using a relative isoperimetric inequality (see, for example, (2.1) below), the coarea formula, the Hardy-Littlewood inequality for rearrangements (see, for example, (1.11) below), a differential inequality involving integrals over the level sets of Sobolev functions established by Maz'ya [19] (see also Lemma 2.1 below) and two boundedness estimates for Schrödinger equations obtained by Shen [24, 25], we show Theorems 1.2-1.5.

Remark 1.6. Let $n \geq 3$ and $0 \leq V \in R H_{n}\left(\mathbb{R}^{n}\right)$ with $V \not \equiv 0$ on $\Omega$.

(i) $\partial \Omega \in W^{2} L^{n-1,1}$ means that $\Omega$ is locally the subgraph of a function of $n-1$ variables whose second order derivatives belong to the Lorentz space $L^{n-1,1}$. It is worth pointing out that $\partial \Omega \in W^{2} L^{n-1,1}$ is the weakest possible integrability condition on second order derivatives for the first order derivatives to be continuous, and hence $\partial \Omega \in C^{1,0}$ (see, for example, [9]).

(ii) The assumption $f \in L^{n, 1}(\Omega)$ in Theorems 1.2-1.5 is sharp. We explain this in the case of Dirichlet boundary problems. Let $B_{0}:=B(0,1)$ be the unit ball centered at 0 . The Poisson equation on $B_{0}$ with the Dirichlet boundary condition is as follows:

$$
\begin{cases}-\Delta v=f & \text { in } B_{0} \\ v=0 & \text { on } \partial B_{0}\end{cases}
$$


It was proved in $\left[3\right.$, p. 512] that $\nabla v \in L^{\infty}\left(B_{0}\right)$ if and only if $f \in L^{n, 1}\left(B_{0}\right)$. Let $c_{0}$ be a positive constant. Then $c_{0} \in R H_{n}\left(\mathbb{R}^{n}\right)$. For the Schrödinger equation $-\Delta u+c_{0} u=f$ on $B_{0}$ with the Dirichlet boundary condition, we see that, when $c_{0}$ is small enough, $\nabla u \in L^{\infty}\left(B_{0}\right)$ if and only if $f \in L^{n, 1}\left(B_{0}\right)$.

In the remainder of this paper, we recall the definitions of the semi-convex domain in $\mathbb{R}^{n}$ and Lorentz(-Sobolev) space.

Definition 1.7. (i) Let $\Omega$ be an open set in $\mathbb{R}^{n}$. The collection of semi-convex functions on $\Omega$ consists of continuous functions $u: \Omega \rightarrow \mathbb{R}$ with the property that there exists a positive constant $C$ such that, for all $x, h \in \mathbb{R}^{n}$ with the ball $B(x,|h|) \subset \Omega$,

$$
2 u(x)-u(x+h)-u(x-h) \leq C|h|^{2} .
$$

The best constant $C$ above is referred as the semi-convexity constant of $u$.

(ii) A nonempty, proper open subset $\Omega$ of $\mathbb{R}^{n}$ is said to be semi-convex provided that there exist $b, c \in(0, \infty)$ with the property that, for every $x_{0} \in \partial \Omega$, there exist an $(n-1)$ dimensional affine variety $H \subset \mathbb{R}^{n}$ passing through $x_{0}$, a choice $N$ of the unit normal to $H$, and an open set

$$
\mathcal{C}:=\left\{\widetilde{x}+t N: \widetilde{x} \in H,\left|\widetilde{x}-x_{0}\right|<b,|t|<c\right\}
$$

(called a coordinate cylinder near $x_{0}$ with axis along $N$ ) satisfying, for some semi-convex function $\varphi: H \rightarrow \mathbb{R}$,

$$
\begin{aligned}
& \mathcal{C} \cap \Omega=\mathcal{C} \cap\{\widetilde{x}+t N: \widetilde{x} \in H, t>\varphi(\widetilde{x})\}, \\
& \mathcal{C} \cap \partial \Omega=\mathcal{C} \cap\{\widetilde{x}+t N: \widetilde{x} \in H, t=\varphi(\widetilde{x})\}, \\
& \mathcal{C} \cap \widetilde{\Omega}^{\complement}=\mathcal{C} \cap\{\widetilde{x}+t N: \widetilde{x} \in H, t<\varphi(\widetilde{x})\}, \\
& \varphi\left(x_{0}\right)=0 \text { and }|\varphi(\widetilde{x})|<c / 2 \text { if }\left|\widetilde{x}-x_{0}\right| \leq b,
\end{aligned}
$$

where $\bar{\Omega}$ and $\bar{\Omega}^{\complement}$, respectively, denotes the closure of $\Omega$ in $\mathbb{R}^{n}$ and the complementary set of $\bar{\Omega}$ in $\mathbb{R}^{n}$.

To characterize the semi-convex domain, we need the following notion.

Definition 1.8. A set $E \subset \mathbb{R}^{n}$ is said to satisfy an exterior ball condition at $x \in \partial E$, if there exist $v \in S^{n-1}$ and $r \in(0, \infty)$ such that

$$
B(x+r v, r) \subset \mathbb{R}^{n} \backslash E,
$$

where $S^{n-1}$ denotes the unit sphere in $\mathbb{R}^{n}$. For such $x \in \partial E$, let

$$
r(x):=\sup \left\{r \in(0, \infty):(1.7) \text { holds true for some } v \in S^{n-1}\right\} .
$$

It is said that the set $E$ satisfies a uniform exterior ball condition (for simplicity, UEBC) with radius $r \in(0, \infty]$, if

$$
\inf _{x \in \partial E} r(x) \geq r
$$

and the value $r$ in (1.8) is referred to the UEBC constant. Then it is said that the set $E$ satisfies a UEBC, if there exists $r \in(0, \infty]$ with the property that $E$ satisfies the uniform exterior ball condition with radius $r$. Moreover, the largest such constant $r$ is called the uniform ball constant of $E$. 
Remark 1.9. (i) It is well known that bounded semi-convex domains in $\mathbb{R}^{n}$ are bounded Lipschitz domains, and the convex domains in $\mathbb{R}^{n}$ are semi-convex domains (see, for example, [20, 22]).

(ii) It is well known that if $\Omega \subset \mathbb{R}^{n}$ is convex, then $\Omega$ satisfies a UEBC with the uniform ball constant $\infty$ (see, for example, [11]). Moreover, for any open set $\Omega \subset \mathbb{R}^{n}$ with compact boundary, it was proved in [21] that $\Omega$ is a Lipschitz domain satisfying a UEBC if and only if $\Omega$ is a semi-convex domain in $\mathbb{R}^{n}$ (see also [20, Theorem 2.5]).

Now we recall the definitions of Lorentz and Lorentz-Sobolev spaces.

Let $\Omega \subset \mathbb{R}^{n}$ be an open bounded set and $u$ be a real-valued measurable function on $\Omega$. Then the distribution function $\mu_{u}:[0, \infty) \rightarrow[0,|\Omega|]$ of $u$ is defined by, for any $t \in[0, \infty)$,

$$
\mu_{u}(t):=|\{x \in \Omega:|u(x)|>t\}| .
$$

The decreasing rearrangement $u^{*}:[0, \infty) \rightarrow[0, \infty]$ of $u$ is defined by, for any $s \in[0, \infty)$,

$$
u^{*}(s):=\sup \left\{t \in[0, \infty): \mu_{u}(t)>s\right\} .
$$

We remark that $u^{*}$ is the unique right-continuous non-increasing function in $[0, \infty)$ equivalently distributed with $u$, and $u^{*}(s)=0$ if $s \geq|\Omega|$. Moreover, the function $u^{* *}:(0, \infty) \rightarrow$ $[0, \infty)$ is defined by, for any $s \in(0, \infty)$,

$$
u^{* *}(s):=\frac{1}{s} \int_{0}^{s} u^{*}(r) d r .
$$

Then it is easy to see that, for any $s \in(0, \infty), u^{*}(s) \leq u^{* *}(s)$. Furthermore, the HardyLittlewood inequality states that, for any measurable functions $u$ and $v$ on $\Omega$,

$$
\int_{\Omega}|u(x) v(x)| d x \leq \int_{0}^{\infty} u^{*}(s) v^{*}(s) d s .
$$

Let $q \in(1, \infty)$ and $s \in(0, \infty]$. Then the Lorentz space $L^{q, s}(\Omega)$ is defined to be all measurable functions $u: \Omega \rightarrow \mathbb{R}$ satisfying that

$$
\|u\|_{L^{q, s}(\Omega)}:=\left\{\int_{0}^{|\Omega|}\left[t^{\frac{1}{q}-\frac{1}{s}} u^{*}(t)\right]^{s} d t\right\}^{1 / s}<\infty .
$$

We remark that, if $s \in[1, \infty], L^{q, s}(\Omega)$ is a Banach space, equipped with the norm, which is equivalent to $\|\cdot\|_{L^{q, s}(\Omega)}$ and is obtained via replacing $u^{*}$ with $u^{* *}$ in (1.12). For Lorentz spaces, we have the following facts:

(i) for $q \in(1, \infty), L^{q, q}(\Omega)=L^{q}(\Omega)$;

(ii) if $q \in(1, \infty)$ and $s_{1}, s_{2} \in[1, \infty]$ with $s_{1}<s_{2}$, then $L^{q, s_{1}}(\Omega) \varsubsetneqq L^{q, s_{2}}(\Omega)$;

(iii) if $q_{1}, q_{2} \in(1, \infty)$ with $q_{1}>q_{2}$ and $s_{1}, s_{2} \in(0, \infty]$, then $L^{q_{1}, s_{1}}(\Omega) \varsubsetneqq L^{q_{2}, s_{2}}(\Omega)$.

Denote by $q^{\prime}$ and $s^{\prime}$ the Hölder's conjugate exponents of $q$ and $s$. Then Hölder's inequality in Lorentz spaces states that there exists a positive constant $C=C_{(q, s)}$, depending on $q$ and $s$, such that, for all $u \in L^{q, s}(\Omega)$ and $v \in L^{q^{\prime}}, s^{\prime}(\Omega)$,

$$
\int_{\Omega}|u(x) v(x)| d x \leq C\|u\|_{L^{q, s}(\Omega)}\|v\|_{L^{q^{\prime}, s^{\prime}}(\Omega)} .
$$


Let $m \in \mathbb{N}, q \in(1, \infty)$ and $s \in[1, \infty]$. The Lorentz-Sobolev space $W^{m} L^{q, s}(\Omega)$ is defined by

$$
\begin{aligned}
W^{m} L^{q, s}(\Omega):= & \left\{u \in L^{q, s}(\Omega): u \text { is } m\right. \text {-times weakly differentiable in } \\
& \left.\Omega \text { and }\left|\nabla^{k} u\right| \in L^{q, s}(\Omega), 1 \leq k \leq m\right\}
\end{aligned}
$$

with the norm

$$
\|u\|_{W^{m} L^{q, s}(\Omega)}:=\|u\|_{L^{q, s}(\Omega)}+\sum_{k=1}^{m}\left\|\left|\nabla^{k} u\right|\right\|_{L^{q, s}(\Omega)} .
$$

Finally we make some conventions on notation. Throughout the whole paper, we always denote by $C$ a positive constant which is independent of the main parameters, but it may vary from line to line. We also use $C_{(\gamma, \beta, \ldots)}$ to denote a positive constant depending on the indicated parameters $\gamma, \beta, \ldots$ The symbol $A \lesssim B$ means that $A \leq C B$. If $A \lesssim B$ and $B \lesssim A$, then we write $A \sim B$. For any measurable subset $E$ of $\mathbb{R}^{n}$, we denote by $\chi_{E}$ its characteristic function. We also let $\mathbb{N}:=\{1,2, \ldots\}$ and $\mathbb{Z}_{+}:=\mathbb{N} \cup\{0\}$. Finally, for $q \in[1, \infty]$, we denote by $q^{\prime}$ its conjugate exponent, namely, $1 / q+1 / q^{\prime}=1$.

\section{Preliminaries}

In this section, we recall some necessary notations and auxiliary conclusions. We first recall a relative isoperimetric inequality. Let $\Omega$ be a bounded Lipschitz domain in $\mathbb{R}^{n}$ with $n \geq 2$. Then there exists a positive constant $C$ such that, for any measurable set $E \subset \Omega$ satisfying $|E| \leq|\Omega| / 2$,

$$
|E|^{1 / n^{\prime}} \leq C \mathcal{H}^{n-1}(\partial E \cap \Omega),
$$

where and in what follows, $\partial E$ denotes the essential boundary of $E$, and $\mathcal{H}^{n-1}$ stands for $(n-1)$-dimensional Hausdorff measure (see [16, Corollary 5.2.1/3] for the details). We point out that the isoperimetric inequality (2.1) can be obtained via the geometric inequality that there exists a positive constant $C=C_{(\Omega)}$ such that, for any measurable set $E \subset \mathbb{R}^{n}$ with $|E| \leq|\Omega| / 2$,

$$
\mathcal{H}^{n-1}(\partial E \cap \partial \Omega) \leq C \mathcal{H}^{n-1}(\partial E \cap \Omega)
$$

(see [16, Chapter 6] for the details) and the classical isoperimetric inequality in $\mathbb{R}^{n}$ that, there exists a positive constant $C=C_{(n)}$ such that, for any measurable set $E \subset \mathbb{R}^{n}$ with $|E|<\infty$

$$
|E|^{1 / n^{\prime}} \leq C \mathcal{H}^{n-1}(\partial E)
$$

We also remark that the constant in (2.1) depends on $n$ and the constant in (2.2) (see, [6, Section 5] or [8, Section 3] for further details about (2.1)).

Let $u \in W^{2,1}(\Omega)$. Then $|\nabla u| \in W^{1,1}(\Omega)$. From an application of the coarea formula for Sobolev functions, it follows that, for any Borel function $g: \Omega \rightarrow[0, \infty)$ and $t \in[0, \infty)$,

$$
\int_{\{|\nabla u|>t\}} g(x)|\nabla| \nabla u(x)|| d x=\int_{t}^{\infty} \int_{\{|\nabla u|=\tau\}} g(x) d \mathcal{H}^{n-1} d \tau,
$$


where $\{|\nabla u|>t\}:=\{x \in \Omega:|\nabla u(x)|>t\}$ and $\{|\nabla u|=t\}:=\{x \in \Omega:|\nabla u(x)|=t\}$ (see, for example, [2]). Moreover, if the left hand side of $(2.3)$ is finite for $t \in(0, \infty)$, then the left hand side of (2.3) is an absolutely continuous function of $t$ and for almost every $t \in(0, \infty)$,

$$
-\frac{d}{d t} \int_{\{|\nabla u|>t\}} g(x)|\nabla| \nabla u(x)|| d x=\int_{\{|\nabla u|=t\}} g(x) d \mathcal{H}^{n-1} .
$$

Furthermore, by the coarea formula again, we know that, for almost every $t \in(0, \infty)$, $\mathcal{H}^{n-1}(\{|\nabla u|=t\} \cap\{|\nabla| \nabla u||=0\})=0$. Thus, for such function $g$ as in (2.3) and $t \in[0, \infty)$,

$$
\int_{\{|\nabla u|>t\}} g(x) d x=\int_{\{|\nabla u|>t\} \cap\{|\nabla| \nabla u||=0\}} g(x) d x+\int_{t}^{\infty} \int_{\{|\nabla u|=\tau\}} \frac{g(x)}{|\nabla| \nabla u(x)||} \mathcal{H}^{n-1} d \tau,
$$

which further implies that, if $g \in L^{1}(\Omega)$, then for almost every $t \in(0, \infty)$,

$$
-\frac{d}{d t} \int_{\{|\nabla u|>t\}} g(x) d x \geq \int_{\{|\nabla u|=t\}} \frac{g(x)}{|\nabla| \nabla u(x)||} \mathcal{H}^{n-1} .
$$

Lemma 2.1. Let $n \geq 2$ and $\Omega$ be a bounded Lipschitz domain in $\mathbb{R}^{n}$. Assume that $v \in W^{1,2}(\Omega)$ is non-negative, $\mu_{v}$ and $v^{*}$ denote the distribution function and the decreasing rearrangement of $v$ defined as in (1.9) and (1.10), respectively. Then there exists a positive constant $C$, depending on the constant in (2.2), such that, for almost every $t \in\left[v^{*}(|\Omega| / 2), \infty\right)$,

$$
1 \leq C\left[-\mu_{v}^{\prime}(t)\right]^{1 / 2}\left[\mu_{v}(t)\right]^{-1 / n^{\prime}}\left\{-\frac{d}{d t} \int_{\{v>t\}}|\nabla v(x)|^{2} d x\right\}^{1 / 2},
$$

where $\mu_{v}^{\prime}$ denotes the derivative of $\mu_{v}$.

Lemma 2.1 was established by Maz'ya [19].

The following Lemmas 2.2, 2.3 and 2.4 are, respectively, just [8, Proposition 3.4, Lemmas 3.5 and 3.6].

Lemma 2.2. Let $\Omega \subset \mathbb{R}^{n}$ be a measurable set, $w: \Omega \rightarrow[0, \infty)$ a measurable function and $g \in L^{1}(\Omega)$. The function $\varphi:(0,|\Omega|) \rightarrow[0, \infty)$ is defined by, for any $s \in(0,|\Omega|)$,

$$
\varphi(s):=\frac{d}{d s} \int_{\left\{w>w^{*}(s)\right\}}|g(x)| d x .
$$

Then for any $s \in(0,|\Omega|)$,

$$
\int_{0}^{s} \varphi^{*}(r) d r \leq \int_{0}^{s} g^{*}(r) d r .
$$

Lemma 2.3. Let $L \in(0, \infty]$ and $\varphi, \psi:[0, L) \rightarrow[0, \infty)$ be measurable functions satisfying that, for any $s \in(0,|\Omega|), \int_{0}^{s}\left[\varphi^{*}(r)\right]^{2} d r \leq \int_{0}^{s}\left[\psi^{*}(r)\right]^{2} d r$. Then for any $\gamma \in(1 / 2, \infty)$, there exists a positive constant $C_{(\gamma)}$, depending on $\gamma$, such that

$$
\int_{0}^{L} \varphi(s) s^{-\gamma} d s \leq C_{(\gamma)} \int_{0}^{L} \psi^{*}(s) s^{-\gamma} d s
$$


Lemma 2.4. Let $L \in(0, \infty]$ and $\gamma \in(1 / 2,1)$. Then there exists a positive constant $C_{(\gamma)}$, depending on $\gamma$, such that, for any non-increasing function $\varphi:(0, L) \rightarrow[0, \infty)$,

$$
\left\{\int_{0}^{L} s^{-2 \gamma} \int_{0}^{s}[\varphi(r)]^{2} d r d s\right\}^{1 / 2} \leq C_{(\gamma)} \int_{0}^{L} s^{-\gamma} \varphi(s) d s .
$$

Lemma 2.5. Let $n \geq 2, \Omega$ be an open set in $\mathbb{R}^{n}$ and $u \in C^{3}(\Omega)$. Then

$$
(\Delta u)^{2}=\operatorname{div}(\Delta u \nabla u)-\sum_{i, j=1}^{n}\left(u_{x_{i} x_{j}} u_{x_{i}}\right)_{x_{j}}+\left|\nabla^{2} u\right|^{2} .
$$

Lemma 2.5 is a corollary of the divergence theorem, the details being omitted here.

Moreover, we need the following properties of the distribution function and the decreasing rearrangement (see, for example, [14, Proposition 1.4.5]).

Lemma 2.6. Let $\Omega$ be an open set in $\mathbb{R}^{n}$ and $f$ a measurable function on $\Omega$. Then for any $t \in[0, \infty)$ and $s \in(0, \infty), \mu_{f}\left(f^{*}(t)\right) \leq t$ and $f^{*}\left(\mu_{f}(s)\right) \leq s$.

Let $\Omega$ be a bounded semi-convex domain in $\mathbb{R}^{n}$ with $C^{2}$ boundary. Denote by $W$ the Weingarten matrix of $\partial \Omega$, which is defined by the requirement that its entries are the coefficients of the second fundamental form of the surface $\partial \Omega$. Following [20], in this paper, $W$ is defined by

$$
W:=\left(\left(\nabla_{T} \nu_{k}\right)_{j}\right)_{1 \leq j, k \leq n},
$$

where and in what follows, $\nabla_{T}$ stands for the tangential gradient, which is defined by $\nabla_{T}:=\nabla-\nu \cdot \nabla$. Then we have the following lemma for bounded semi-convex domains in $\mathbb{R}^{n}$, which was established in [21] (see also [20, Theorem 2.6]).

Lemma 2.7. Let $n \geq 2$ and $\Omega$ be a bounded domain in $\mathbb{R}^{n}$ with $C^{2}$ boundary, in particular, a Lipschitz domain satisfying $U E B C$ with some constant $r_{0} \in(0, \infty]$. Then the Weingarten matrix of $\partial \Omega$ is bounded from below by $-C_{1} / r_{0}$ for almost every point on $\partial \Omega$ with respect to the measure $\mathcal{H}^{n-1}$, where the positive constant $C_{1}$ depend only on the Lipschitz character of $\Omega$.

Lemma 2.8. Let $n \geq 3$ and $\Omega \subset \mathbb{R}^{n}$ be a bounded domain with $\partial \Omega \in C^{2}$. Assume that $u \in C^{\infty}(\Omega) \cap C^{2}(\bar{\Omega})$ satisfies $u=0$ on $\partial \Omega$. Let $\mathcal{B}$ denote the second fundamental form on $\partial \Omega$ and $\operatorname{tr} \mathcal{B}$ be its trace. Then for almost every $t \in(0, \infty)$,

$$
\begin{aligned}
t \int_{\{|\nabla u|=t\}}|\nabla| \nabla u(x)|| d \mathcal{H}^{n-1} \leq & t \int_{\{|\nabla u|=t\}}|\Delta u(x)| d \mathcal{H}^{n-1}+\int_{\{|\nabla u|>t\}}|\Delta u(x)|^{2} d x \\
& +\|\nabla u\|_{L^{\infty}(\Omega)}^{2} \int_{\partial \Omega \cap \partial\{|\nabla u|>t\}}|\operatorname{tr} \mathcal{B}(x)| d \mathcal{H}^{n-1} .
\end{aligned}
$$

Moreover, if $r \in(n-1, \infty)$, then for almost every $t \in\left[t_{u}, \infty\right)$,

$$
\begin{aligned}
t \int_{\{|\nabla u|=t\}}|\nabla| \nabla u(x)|| d \mathcal{H}^{n-1} \leq & t \int_{\{|\nabla u|=t\}}|\Delta u(x)| d \mathcal{H}^{n-1}+\int_{\{|\nabla u|>t\}}|\Delta u(x)|^{2} d x \\
& +2 t^{2} \int_{\partial \Omega \cap \partial\{|\nabla u|>t\}}|\operatorname{tr} \mathcal{B}(x)| d \mathcal{H}^{n-1},
\end{aligned}
$$


where $t_{u}:=|\nabla u|^{*}(\alpha \Omega)$ with $\alpha \in(0,1 / 2]$ being a constant depending on $n, r,\|\operatorname{tr} \mathcal{B}\|_{L^{r}(\partial \Omega)}$, $\Omega$ and the constant in (2.2).

If $\Omega$ is semi-convex, then there exists a positive constant $C$, depending on the Lipschitz character and the uniform ball constant of $\Omega$, such that, for almost every $t \in(0, \infty)$,

$$
\begin{aligned}
t \int_{\{|\nabla u|=t\}}|\nabla| \nabla u(x)|| d \mathcal{H}^{n-1} \leq & t \int_{\{|\nabla u|=t\}}|\Delta u(x)| d \mathcal{H}^{n-1}+\int_{\{|\nabla u|>t\}}|\Delta u(x)|^{2} d x \\
& +C\|\nabla u\|_{L^{\infty}(\Omega)}^{2} \mathcal{H}^{n-1}(\partial \Omega \cap \partial\{|\nabla u|>t\}) .
\end{aligned}
$$

Furthermore, there exist positive constants $\alpha \in(0,1 / 2]$, depending on $n, \Omega$ and the constant in (2.2), and $C$, depending on the Lipschitz character and the uniform ball constant of $\Omega$, such that, for almost every $t \in\left[t_{u}, \infty\right)$, where $t_{u}:=|\nabla u|^{*}(\alpha \Omega)$,

$$
\begin{aligned}
t \int_{\{|\nabla u|=t\}}|\nabla| \nabla u(x)|| d \mathcal{H}^{n-1} \leq & t \int_{\{|\nabla u|=t\}}|\Delta u(x)| d \mathcal{H}^{n-1}+\int_{\{|\nabla u|>t\}}|\Delta u(x)|^{2} d x \\
& +C t^{2} \mathcal{H}^{n-1}(\partial \Omega \cap \partial\{|\nabla u|>t\}) .
\end{aligned}
$$

To prove Lemma 2.8, we need the following conclusion, which is just [6, Lemma 5.1].

Lemma 2.9. Let $n \geq 3, \Omega \subset \mathbb{R}^{n}$ be a bounded Lipschitz domain and $q \in[1,2(n-1) /(n-$ $2)$. Then there exists a positive constant $C$, depending on $n, q$ and the constant in $(2.2)$, such that, for any $u \in W^{1,2}(\Omega)$ with $|\operatorname{supp} u| \leq|\Omega| / 2$,

$$
\left\{\int_{\partial \Omega}|\operatorname{Tr} u(x)|^{q} d \mathcal{H}^{n-1}\right\}^{1 / q} \leq C\left\{\int_{\Omega}|\nabla u(x)|^{n q /(n+q-1)} d x\right\}^{(n+q-1) / n q} .
$$

Now we prove Lemma 2.8 by using Lemmas $2.5,2.6,2.7$ and 2.9.

Proof of Lemma 2.8. To finish the proof of Lemma 2.8, we borrow some ideas from the proof of $[6$, Lemma 5.4]. It is easy to see that, for all $t \in(0, \infty)$, the level set $\{|\nabla u|>t\}$ is open and

$$
\partial\{|\nabla u|>t\}=\{|\nabla u|=t\} \cup(\partial \Omega \cap \partial\{|\nabla u|>t\}) .
$$

Moreover, by Lemma 2.5 and the divergence theorem, we conclude that, for almost every $t \in(0, \infty)$,

$$
\begin{aligned}
& \int_{\{|\nabla u|>t\}}[\Delta u(x)]^{2} d x \\
& =\int_{\{|\nabla u|>t\}} \operatorname{div}(\Delta u(x) \nabla u(x)) d x-\int_{\{|\nabla u|>t\}} \sum_{i, j=1}^{n}\left(u_{x_{i} x_{j}}(x) u_{x_{i}}(x)\right)_{x_{j}} d x \\
& \quad+\int_{\{|\nabla u|>t\}}\left|\nabla^{2} u(x)\right|^{2} d x \\
& =\int_{\partial\{|\nabla u|>t\}} \Delta u(x) \frac{\partial u(x)}{\partial \nu} d \mathcal{H}^{n-1}-\int_{\partial\{|\nabla u|>t\}} \sum_{i, j=1}^{n} u_{x_{i} x_{j}}(x) u_{x_{i}}(x) \nu_{j} d \mathcal{H}^{n-1} \\
& \quad+\int_{\{|\nabla u|>t\}}\left|\nabla^{2} u(x)\right|^{2} d x
\end{aligned}
$$


which, combined with $(2.11)$ and the fact that, for almost every $t \in(0, \infty)$,

$$
\nu=-\frac{\nabla|\nabla u|}{|\nabla| \nabla u||} \quad \text { on }\{|\nabla u|=t\}
$$

and $\sum_{i=1}^{n} u_{x_{i} x_{j}} u_{x_{i}}=|\nabla u|_{x_{j}}|\nabla u|$, further implies that

$$
\begin{aligned}
& \int_{\partial\{|\nabla u|>t\}} \Delta u(x) \frac{\partial u(x)}{\partial \nu} d \mathcal{H}^{n-1}-\int_{\partial\{|\nabla u|>t\}} \sum_{i, j=1}^{n} u_{x_{i} x_{j}}(x) u_{x_{i}}(x) \nu_{j} d \mathcal{H}^{n-1} \\
& =\int_{\{|\nabla u|=t\}} \Delta u(x) \frac{\partial u(x)}{\partial \nu} d \mathcal{H}^{n-1}+t \int_{\{|\nabla u|=t\}}|\nabla| \nabla u(x)|| d \mathcal{H}^{n-1} \\
& \quad+\int_{\partial \Omega \cap \partial\{|\nabla u|>t\}}\left[\Delta u(x) \frac{\partial u(x)}{\partial \nu}-\sum_{i, j=1}^{n} u_{x_{i} x_{j}}(x) u_{x_{i}}(x) \nu_{j}\right] d \mathcal{H}^{n-1}
\end{aligned}
$$

Furthermore, from $[15,(3.1 .1 .8)]$, it follows that on $\partial \Omega$,

$$
\begin{aligned}
\Delta u \frac{\partial u}{\partial \nu}-\sum_{i, j=1}^{n} u_{x_{i} x_{j}} u_{x_{i}} \nu_{j}= & \operatorname{div}_{T}\left(\frac{\partial u}{\partial \nu} \nabla_{T} u\right)-\operatorname{tr} \mathcal{B}\left(\frac{\partial u}{\partial \nu}\right)^{2} \\
& -\mathcal{B}\left(\nabla_{T} u, \nabla_{T} u\right)-2 \nabla_{T} u \cdot \nabla_{T} \frac{\partial u}{\partial \nu}
\end{aligned}
$$

where $\operatorname{div}_{T}$ and $\nabla_{T}$ denote the divergence operator and the gradient operator on $\partial \Omega$, which, together with the assumption $u=0$ on $\partial \Omega$, implies that on $\partial \Omega$,

$$
\Delta u \frac{\partial u}{\partial \nu}-\sum_{i, j=1}^{n} u_{x_{i} x_{j}} u_{x_{i}} \nu_{j}=-\operatorname{tr} \mathcal{B}\left(\frac{\partial u}{\partial \nu}\right)^{2}
$$

By this, we find that, for almost every $t \in(0, \infty)$,

$$
\begin{aligned}
& \int_{\partial \Omega \cap \partial\{|\nabla u|>t\}}\left[\Delta u(x) \frac{\partial u(x)}{\partial \nu}-\sum_{i, j=1}^{n} u_{x_{i} x_{j}}(x) u_{x_{i}}(x) \nu_{j}\right] d \mathcal{H}^{n-1} \\
& \geq-\int_{\partial \Omega \cap \partial\{|\nabla u|>t\}}|\nabla u(x)|^{2}|\operatorname{tr} \mathcal{B}(x)| d \mathcal{H}^{n-1}
\end{aligned}
$$

Moreover, it is easy to see that

$$
\left|\int_{\{|\nabla u|=t\}} \Delta u(x) \frac{\partial u(x)}{\partial \nu} d \mathcal{H}^{n-1}\right| \leq t \int_{\{|\nabla u|=t\}}|\Delta u(x)| d \mathcal{H}^{n-1},
$$

which, combined with (2.12), (2.13), (2.16) and (2.17), further implies that, for almost every $t \in(0, \infty)$,

$$
t \int_{\{|\nabla u|=t\}}|\nabla| \nabla u(x)|| d \mathcal{H}^{n-1}+\int_{\{|\nabla u|>t\}}\left|\nabla^{2} u(x)\right|^{2} d x
$$


Global Boundedness of the Gradient for a Class of Schrödinger Equations

$$
\begin{aligned}
\leq & t \int_{\{|\nabla u|=t\}}|\Delta u(x)| d \mathcal{H}^{n-1}+\int_{\{|\nabla u|>t\}}|\Delta u(x)|^{2} d x \\
& +\int_{\partial \Omega \cap \partial\{|\nabla u|>t\}}|\nabla u(x)|^{2}|\operatorname{tr} \mathcal{B}(x)| d \mathcal{H}^{n-1} .
\end{aligned}
$$

By this, we conclude that (2.7) holds true.

Now we prove (2.8). From Hölder's inequality, we deduce that, for all $t \in(0, \infty)$,

$$
\begin{aligned}
& \int_{\partial \Omega \cap \partial\{|\nabla u|>t\}}|\nabla u(x)|^{2}|\operatorname{tr} \mathcal{B}(x)| d \mathcal{H}^{n-1} \\
& \leq 2 t^{2} \int_{\partial \Omega \cap \partial\{|\nabla u|>t\}}|\operatorname{tr} \mathcal{B}(x)| d \mathcal{H}^{n-1} \\
& \quad+2 \int_{\partial \Omega \cap \partial\{|\nabla u|>t\}}(|\nabla u(x)|-t)^{2}|\operatorname{tr} \mathcal{B}(x)| d \mathcal{H}^{n-1} .
\end{aligned}
$$

For simplicity, denote by $\mu$ the distribution function $\mu_{|\nabla u|}$ of $|\nabla u|$. Let $\delta:=(n-1) / n r^{\prime}-$ $(n-2) / n$. By $r>n-1$, we know that $\delta>0$, which, together with the facts $\max \{|\nabla u|-$ $t, 0\} \in W^{1,2}(\Omega)$ and $|\nabla| \nabla u|| \leq\left|\nabla^{2} u\right|$, Hölder's inequality and Lemma 2.9, implies that, for any $t \in\left[|\nabla u|^{*}(|\Omega| / 2), \infty\right)$,

$$
\begin{aligned}
& \int_{\partial \Omega \cap \partial\{|\nabla u|>t\}}(|\nabla u(x)|-t)^{2}|\operatorname{tr} \mathcal{B}(x)| d \mathcal{H}^{n-1} \\
& \quad \leq\left[\int_{\partial \Omega \cap \partial\{|\nabla u|>t\}}(|\nabla u(x)|-t)^{2 r^{\prime}} d \mathcal{H}^{n-1}\right]^{1 / r^{\prime}}\left[\int_{\partial \Omega \cap \partial\{|\nabla u|>t\}}|\operatorname{tr} \mathcal{B}(x)|^{r} d \mathcal{H}^{n-1}\right]^{1 / r} \\
& \quad \leq C_{2}[\mu(t)]^{\delta}\|\operatorname{tr} \mathcal{B}\|_{L^{r}(\partial \Omega)} \int_{\{|\nabla u|>t\}}\left|\nabla^{2} u(x)\right|^{2} d x
\end{aligned}
$$

where $C_{2}$ is a positive constant depending on $r$ and the constant in (2.2). Let

$$
\beta:=\left[\frac{1}{2 C_{2}\|\operatorname{tr} \mathcal{B}\|_{L^{r}(\partial \Omega)}}\right]^{1 / \delta},
$$

$\alpha:=\min \{\beta /|\Omega|, 1 / 2\}$ and $t_{u}:=|\nabla u|^{*}(\alpha|\Omega|)$. Then it follows, from Lemma 2.6, that, for any $t \in\left[t_{u}, \infty\right)$,

$$
1-2 C_{2}[\mu(t)]^{\delta}\|\operatorname{tr} \mathcal{B}\|_{L^{r}(\partial \Omega)} \geq 0
$$

which, combined with (2.17), (2.18) and (2.19), further implies that (2.8) holds true.

Now we prove (2.9). For any $x \in \Omega$, denote by $T_{x} \partial \Omega$ the $(n-1)$-dimensional tangent plane to $\partial \Omega$ at $x$. Recall that, for any $x \in \partial \Omega$, the second fundamental form $\mathcal{B}(x)$ of $\partial \Omega$ at $x$ is the bilinear map on $T_{x} \partial \Omega \times T_{x} \partial \Omega$ given by, for any $\xi, \eta \in T_{x} \partial \Omega$,

$$
-\mathcal{B}(x)(\xi, \eta):=\left(\nabla_{T} v(x) \xi\right) \cdot \eta,
$$

where $\nabla_{T}$ denote the gradient operator on $\partial \Omega$. Extend the bilinear map in (2.20) to $\mathbb{R}^{n} \times \mathbb{R}^{n}$ by demanding that a pair of vectors $(\xi, \eta)$ is mapped to zero if any of them is 
normal. Then as pointed in [20, Definition 2.3], the Weingarten matrix of $\partial \Omega$ is then the $n \times n$ matrix associated with the extension. Thus, schematically, $W=\nabla_{T} \nu$ on $\partial \Omega$ (with the understanding that the tangential gradient acts on the components of $\nu$ ), which, together with Lemma 2.7 and the definition of $\operatorname{tr} \mathcal{B}$, implies that, there exists a positive constant $C_{3}$, depending on the Lipschitz character and the uniform ball constant of $\Omega$, such that, for all $x \in \partial \Omega, \operatorname{tr} \mathcal{B}(x) \leq C_{3}$, which, combined with (2.15), implies that, for almost every $t \in(0, \infty)$,

$$
\begin{aligned}
& \int_{\partial \Omega \cap \partial\{|\nabla u|>t\}}\left[\Delta u(x) \frac{\partial u(x)}{\partial \nu}-\sum_{i, j=1}^{n} u_{x_{i} x_{j}}(x) u_{x_{i}}(x) \nu_{j}\right] d \mathcal{H}^{n-1} \\
& \geq-C_{3} \int_{\partial \Omega \cap \partial\{|\nabla u|>t\}}|\nabla u(x)|^{2} d \mathcal{H}^{n-1} .
\end{aligned}
$$

By replacing (2.16) with (2.21) and repeating the proof of (2.17), we conclude that, for almost every $t \in(0, \infty)$,

$$
\begin{aligned}
& t \int_{\{|\nabla u|=t\}}|\nabla| \nabla u(x)|| d \mathcal{H}^{n-1}+\int_{\{|\nabla u|>t\}}\left|\nabla^{2} u(x)\right|^{2} d x \\
& \leq t \int_{\{|\nabla u|=t\}}|\Delta u(x)| d \mathcal{H}^{n-1}+\int_{\{|\nabla u|>t\}}|\Delta u(x)|^{2} d x \\
& \quad+C_{3} \int_{\partial \Omega \cap \partial\{|\nabla u|>t\}}|\nabla u(x)|^{2} d \mathcal{H}^{n-1},
\end{aligned}
$$

which further implies that (2.9) holds true.

Finally, we prove (2.10). From Hölder's inequality, we deduce that, for all $t \in(0, \infty)$,

$$
\begin{aligned}
& \int_{\partial \Omega \cap \partial\{|\nabla u|>t\}}|\nabla u(x)|^{2} d \mathcal{H}^{n-1} \\
& \quad \leq 2 \int_{\partial \Omega \cap \partial\{|\nabla u|>t\}}(|\nabla u(x)|-t)^{2} d \mathcal{H}^{n-1}+2 t^{2} \mathcal{H}^{n-1}(\partial \Omega \cap \partial\{|\nabla u|>t\}) .
\end{aligned}
$$

Moreover, repeating the proof of (2.19), we know that, for any $t \in\left[|\nabla u|^{*}(|\Omega| / 2), \infty\right)$,

$$
\begin{aligned}
& \int_{\partial \Omega \cap \partial\{|\nabla u|>t\}}(|\nabla u(x)|-t)^{2} d \mathcal{H}^{n-1} \\
& \leq C_{4}[\mu(t)]^{\delta}\left[\mathcal{H}^{n-1}(\partial \Omega \cap \partial\{|\nabla u|>t\})\right]^{1 / r} \int_{\{|\nabla u|>t\}}\left|\nabla^{2} u(x)\right|^{2} d x
\end{aligned}
$$

where $\delta$ is as in (2.19) and $C_{4}$ is a positive constant depending on $r$ and the constant in (2.2). Taking

$$
\beta:=\left\{\frac{1}{2 C_{4}\left[\mathcal{H}^{n-1}(\partial \Omega)\right]^{1 / r}}\right\}^{1 / \delta},
$$

$\alpha:=\min \{\beta /|\Omega|, 1 / 2\}$ and $t_{u}:=|\nabla u|^{*}(\alpha|\Omega|)$, replacing (2.19) with (2.22) and repeating the proof of $(2.8)$, we conclude that $(2.10)$ holds true. This finishes the proof of Lemma 2.8 . 
Global Boundedness of the Gradient for a Class of Schrödinger Equations

Lemma 2.10. Let $n \geq 3$ and $\Omega \subset \mathbb{R}^{n}$ be a bounded domain with $\partial \Omega \in C^{2}$. Assume that $u \in C^{\infty}(\Omega) \cap C^{2}(\bar{\Omega})$ satisfies $\frac{\partial u}{\partial \nu}=0$ on $\partial \Omega$. For any $x \in \partial \Omega$, let

$$
\widetilde{\mathcal{B}}(x):=\sup _{0 \neq v \in \mathbb{R}^{n-1}} \frac{\mathcal{B}(x)(v, v)}{|v|^{2}} .
$$

Then for almost every $t \in(0, \infty)$,

$$
\begin{aligned}
t \int_{\{|\nabla u|=t\}}|\nabla| \nabla u(x)|| d \mathcal{H}^{n-1} \leq & t \int_{\{|\nabla u|=t\}}|\Delta u(x)| d \mathcal{H}^{n-1}+\int_{\{|\nabla u|>t\}}|\Delta u(x)|^{2} d x \\
& +\|\nabla u\|_{L^{\infty}(\Omega)}^{2} \int_{\partial \Omega \cap \partial\{|\nabla u|>t\}}|\widetilde{\mathcal{B}}(x)| d \mathcal{H}^{n-1} .
\end{aligned}
$$

Moreover, if $r \in(n-1, \infty)$, then for almost every $t \in\left[t_{u}, \infty\right)$,

$$
\begin{aligned}
t \int_{\{|\nabla u|=t\}}|\nabla| \nabla u(x)|| d \mathcal{H}^{n-1} \leq & t \int_{\{|\nabla u|=t\}}|\Delta u(x)| d \mathcal{H}^{n-1}+\int_{\{|\nabla u|>t\}}|\Delta u(x)|^{2} d x \\
& +2 t^{2} \int_{\partial \Omega \cap \partial\{|\nabla u|>t\}}|\widetilde{\mathcal{B}}(x)| d \mathcal{H}^{n-1},
\end{aligned}
$$

where $t_{u}:=|\nabla u|^{*}(\alpha \Omega)$ with $\alpha \in(0,1 / 2]$ being a constant depending on $n, r,\|\widetilde{\mathcal{B}}\|_{L^{r}(\partial \Omega)}, \Omega$ and the constant in (2.2).

If $\Omega$ is semi-convex, then there exists a positive constant $C$, depending on the Lipschitz character and the uniform ball constant of $\Omega$, such that, for almost every $t \in(0, \infty)$,

$$
\begin{aligned}
t \int_{\{|\nabla u|=t\}}|\nabla| \nabla u(x)|| d \mathcal{H}^{n-1} \leq & t \int_{\{|\nabla u|=t\}}|\Delta u(x)| d \mathcal{H}^{n-1}+\int_{\{|\nabla u|>t\}}|\Delta u(x)|^{2} d x \\
& +C\|\nabla u\|_{L^{\infty}(\Omega)}^{2} \mathcal{H}^{n-1}(\partial \Omega \cap \partial\{|\nabla u|>t\}) .
\end{aligned}
$$

Furthermore, there exist positive constants $\alpha \in(0,1 / 2]$, depending on $n, \Omega$ and the constant in (2.2), and $C$, depending on the Lipschitz character and the uniform ball constant of $\Omega$, such that, for almost every $t \in\left[t_{u}, \infty\right)$, where $t_{u}:=|\nabla u|^{*}(\alpha \Omega)$,

$$
\begin{aligned}
t \int_{\{|\nabla u|=t\}}|\nabla| \nabla u(x)|| d \mathcal{H}^{n-1} \leq & t \int_{\{|\nabla u|=t\}}|\Delta u(x)| d \mathcal{H}^{n-1}+\int_{\{|\nabla u|>t\}}|\Delta u(x)|^{2} d x \\
& +C t^{2} \mathcal{H}^{n-1}(\partial \Omega \cap \partial\{|\nabla u|>t\}) .
\end{aligned}
$$

Proof. The proof of this lemma is similar to that of Lemma 2.8. By (2.14) and the assumption that $\frac{\partial u}{\partial \nu}=0$ on $\partial \Omega$, we know that

$$
\Delta u \frac{\partial u}{\partial \nu}-\sum_{i, j=1}^{n} u_{x_{i} x_{j}} u_{x_{i}} \nu_{j}=-\mathcal{B}\left(\nabla_{T} u, \nabla_{T} u\right) .
$$

Replacing (2.15) with (2.27) and repeating the proof of Lemma 2.8, we conclude that Lemma 2.10 holds true. 


\section{Proofs of Theorems 1.2-1.5}

In this section, we give out the proofs of Theorems 1.2-1.5. To finish this, we need the following auxiliary lemma, which is just [14, Theorem 1.4.19].

Lemma 3.1. Let $r \in(0, \infty], p_{0}, p_{1} \in(0, \infty]$ with $p_{0} \neq p_{1}, \Omega \subset \mathbb{R}^{n}$ be a bounded open set and $T$ a linear operator defined on the set of simple functions on $\Omega$ and taking values in the set of measurable functions on $\Omega$. Assume further that there exists positive constants $M_{0}$ and $M_{1}$ such that, for all measurable subsets $E \subset \Omega$,

$$
\left\|T\left(\chi_{E}\right)\right\|_{L^{p_{0}, \infty}(\Omega)} \leq M_{0}|E|^{1 / p_{0}} \text { and }\left\|T\left(\chi_{E}\right)\right\|_{L^{p_{1}, \infty}(\Omega)} \leq M_{1}|E|^{1 / p_{1}} .
$$

For any $\theta \in(0,1)$, let

$$
\frac{1}{p}=\frac{1-\theta}{p_{0}}+\frac{\theta}{p_{1}}
$$

Then there exists a positive constant $C$, depending on $p_{0}, p_{1}, M_{0}, M_{1}, r$ and $\theta$, such that, for all functions $f$ in the domain of $T$ and in $L^{p, r}(\Omega)$,

$$
\|T f\|_{L^{p, r}(\Omega)} \leq C\|f\|_{L^{p, r}(\Omega)} .
$$

We now prove Theorem 1.2 by using Lemma 3.1 .

Proof of Theorem 1.2. To prove Theorem 1.2, we borrow some ideas from the proof of $[6$, Theorem 2.1]. We split the proof of this theorem in the following six steps.

Step 1. We first assume that $\partial \Omega \in C^{\infty}$. By $f \in L^{n, 1}(\Omega)$, we see that $f \in L^{2}(\Omega)$. Then from Remark 1.1(iii), it follows that the weak solution $u$ of $(1.2)$ belongs to $W_{0}^{1,2}(\Omega) \cap$ $W^{2,2}(\Omega)$. By the standard approximation, we know that there exists a sequence $\left\{u_{k}\right\}_{k \in \mathbb{N}} \subset$ $C^{\infty}(\Omega) \cap C^{2}(\bar{\Omega})$ such that, for any $k \in \mathbb{N}, u_{k}=0$ on $\partial \Omega$,

$$
\begin{aligned}
& u_{k} \rightarrow u \text { in } W_{0}^{1,2}(\Omega), \quad u_{k} \rightarrow u \text { in } W^{2,2}(\Omega), \\
& \nabla u_{k} \rightarrow \nabla u \text { almost everywhere in } \Omega,
\end{aligned}
$$

as $k \rightarrow \infty$, which, combined with Hölder's inequality and Sobolev's inequality, further implies that

$$
\begin{aligned}
\left\|\Delta u_{k}-V u_{k}+f\right\|_{L^{2}(\Omega)} & \leq\left\|\Delta\left(u_{k}-u\right)\right\|_{L^{2}(\Omega)}+\left\|V\left(u_{k}-u\right)\right\|_{L^{2}(\Omega)} \\
& \leq\left\|\nabla^{2}\left(u_{k}-u\right)\right\|_{L^{2}(\Omega)}+\|V\|_{L^{n}(\Omega)}\left\|u_{k}-u\right\|_{L^{2 n /(n-2)}(\Omega)} \\
& \lesssim\left\|u_{k}-u\right\|_{W^{2,2}(\Omega)}+\|V\|_{L^{n}(\Omega)}\left\|u_{k}-u\right\|_{W^{1,2}(\Omega)} \rightarrow 0,
\end{aligned}
$$

as $k \rightarrow \infty$. By this, we conclude that

$$
-\Delta u_{k}+V u_{k} \rightarrow f \text { in } L^{2}(\Omega),
$$

as $k \rightarrow \infty$.

Step 2. Let $\left\{u_{k}\right\}_{k \in \mathbb{N}}$ be the sequence as in Step 1. For each $k \in \mathbb{N}, u_{k}$ satisfies the same assumptions as the function $u$ in Lemma 2.8. Thus, from (2.8), we deduce that, for each $k \in \mathbb{N}$,

$$
t \int_{\left\{\left|\nabla u_{k}\right|=t\right\}}|\nabla| \nabla u_{k}(x)|| d \mathcal{H}^{n-1}
$$


Global Boundedness of the Gradient for a Class of Schrödinger Equations

$$
\begin{aligned}
\leq & t \int_{\left\{\left|\nabla u_{k}\right|=t\right\}}\left|\Delta u_{k}(x)\right| d \mathcal{H}^{n-1}+\int_{\left\{\left|\nabla u_{k}\right|>t\right\}}\left|\Delta u_{k}(x)\right|^{2} d x \\
& +2 t^{2} \int_{\partial \Omega \cap \partial\left\{\left|\nabla u_{k}\right|>t\right\}}|\operatorname{tr} \mathcal{B}(x)| d \mathcal{H}^{n-1},
\end{aligned}
$$

where $t_{u_{k}}$ is defined analogously to $t_{u}$ as in Lemma 2.8. By using (3.3) and (3.1), similar to the proof of $[6,(6.16)]$, we obtain that, for almost every $t \in\left(t_{u}, \infty\right)$,

$$
\begin{aligned}
& t \int_{\{|\nabla u|=t\}}|\nabla| \nabla u(x)|| d \mathcal{H}^{n-1} \\
& \quad \leq t \int_{\{|\nabla u|=t\}}|f(x)-V(x) u(x)| d \mathcal{H}^{n-1}+\int_{\{|\nabla u|>t\}}|f(x)-V(x) u(x)|^{2} d x \\
& \quad+2 t^{2} \int_{\partial \Omega \cap \partial\{|\nabla u|>t\}}|\operatorname{tr} \mathcal{B}(x)| d \mathcal{H}^{n-1} .
\end{aligned}
$$

Step 3. In this step, we show that, for any given $r \in(n-1, \infty)$,

$$
\|\nabla u\|_{L^{\infty}(\Omega)} \leq C\|f-V u\|_{L^{n, 1}(\Omega)},
$$

where $C$ is a positive constant depending on $n, r,\|\operatorname{tr} \mathcal{B}\|_{L^{r}(\partial \Omega)}, \Omega$ and the constant in (2.2).

By the Hardy-Littlewood inequality (1.11), we find that, for almost every $t \in(0, \infty)$,

$$
\int_{\partial \Omega \cap \partial\{|\nabla u|>t\}}|\operatorname{tr} \mathcal{B}(x)| d \mathcal{H}^{n-1} \leq \int_{0}^{\mathcal{H}^{n-1}(\partial \Omega \cap \partial\{|\nabla u|>t\})}(\operatorname{tr} \mathcal{B})^{*}(r) d r .
$$

Moreover, it follows, from $|\nabla u| \in W^{1,2}(\Omega)$, that, for almost every $t \in(0, \infty)$,

$$
\Omega \cap \partial\{|\nabla u|>t\}=\{|\nabla u|=t\} \text { up to sets of } \mathcal{H}^{n-1} \text { measure zero }
$$

(see, for example, [2]), which, together with (2.2), implies that, for almost every $t \in$ $\left[|\nabla u|^{*}(|\Omega| / 2), \infty\right)$,

$$
\mathcal{H}^{n-1}(\partial \Omega \cap \partial\{|\nabla u|>t\}) \leq C \mathcal{H}^{n-1}(\{|\nabla u|=t\}),
$$

where $C$ is as in (2.2). Denote simply by $\mu$ the distribution function $\mu_{|\nabla u|}$. Then by (2.1), we conclude that, for almost every $t \in\left[|\nabla u|^{*}(|\Omega| / 2), \infty\right)$,

$$
[\mu(t)]^{1 / n^{\prime}} \leq C \mathcal{H}^{n-1}(\{|\nabla u|=t\}),
$$

which, combined with (3.6) and (3.7), implies that, for almost every $t \in\left[|\nabla u|^{*}(|\Omega| / 2), \infty\right)$,

$$
\begin{aligned}
\int_{\partial \Omega \cap \partial\{|\nabla u|>t\}}|\operatorname{tr} \mathcal{B}(x)| d \mathcal{H}^{n-1} & \leq \int_{0}^{C \mathcal{H}^{n-1}(\{|\nabla u|=t\})}(\operatorname{tr} \mathcal{B})^{*}(r) d r \\
& =C \mathcal{H}^{n-1}(\{|\nabla u|=t\})(\operatorname{tr} \mathcal{B})^{* *}\left(C \mathcal{H}^{n-1}(\{|\nabla u|=t\})\right) \\
& \leq C \mathcal{H}^{n-1}(\{|\nabla u|=t\})(\operatorname{tr} \mathcal{B})^{* *}\left([\mu(t)]^{1 / n^{\prime}}\right) .
\end{aligned}
$$

From this and (3.4), we deduce that, for almost every $t \in\left(t_{u}, \infty\right)$,

$$
t \int_{\{|\nabla u|=t\}}|\nabla| \nabla u(x)|| d \mathcal{H}^{n-1}
$$




$$
\begin{aligned}
\leq & t \int_{\{|\nabla u|=t\}}|f(x)-V(x) u(x)| d \mathcal{H}^{n-1}+\int_{\{|\nabla u|>t\}}|f(x)-V(x) u(x)|^{2} d x \\
& +2 C t^{2} \mathcal{H}^{n-1}(\{|\nabla u|=t\})(\operatorname{tr} \mathcal{B})^{* *}\left([\mu(t)]^{1 / n^{\prime}}\right) .
\end{aligned}
$$

Moreover, by Hölder's inequality, (2.5) and (2.4), we know that, for almost every $t \in(0, \infty)$,

$$
\begin{aligned}
& \int_{\{|\nabla u|=t\}}|f(x)-V(x) u(x)| d \mathcal{H}^{n-1} \\
& \leq\left\{\int_{\{|\nabla u|=t\}} \frac{|f(x)-V(x) u(x)|^{2}}{|\nabla| \nabla u(x)||} d \mathcal{H}^{n-1}\right\}^{1 / 2}\left\{\int_{\{|\nabla u|=t\}}|\nabla| \nabla u(x)|| d \mathcal{H}^{n-1}\right\}^{1 / 2} \\
& \leq\left\{-\frac{d}{d t} \int_{\{|\nabla u|>t\}}|f(x)-V(x) u(x)|^{2} d x\right\}^{1 / 2}\left\{-\left.\frac{d}{d t} \int_{\{|\nabla u|>t\}}|\nabla| \nabla u(x)\right|^{2} d x\right\}^{1 / 2} .
\end{aligned}
$$

An argument similar to (3.9) yields that, for almost every $t \in(0, \infty)$,

$$
\mathcal{H}^{n-1}(\{|\nabla u|=t\}) \leq\left[-\mu^{\prime}(t)\right]^{1 / 2}\left\{-\left.\frac{d}{d t} \int_{\{|\nabla u|>t\}}|\nabla| \nabla u(x)\right|^{2} d x\right\}^{1 / 2} .
$$

Furthermore, from the Hardy-Littlewood inequality (1.11), it follows that

$$
\int_{\{|\nabla u|>t\}}|f(x)-V(x) u(x)|^{2} d x \leq \int_{0}^{\mu(t)}\left[|f-V u|^{*}(r)\right]^{2} d r .
$$

By (2.4), (3.8), (3.9), (3.10), (3.11) and (2.6), we conclude that, for almost every $t \in$ $\left(t_{u}, \infty\right)$,

$$
\begin{aligned}
& t\left[-\left.\frac{d}{d t} \int_{\{|\nabla u|>t\}}|\nabla| \nabla u(x)\right|^{2} d x\right] \\
& \leq t\left[-\frac{d}{d t} \int_{\{|\nabla u|>t\}}|f(x)-V(x) u(x)|^{2} d x\right]^{1 / 2}\left[-\frac{d}{d t} \int_{\{|\nabla u|>t\}}|\nabla| \nabla u(x) \|^{2} d x\right]^{1 / 2} \\
& \quad+\left[-\mu^{\prime}(t)\right]^{1 / 2}[\mu(t)]^{-1 / n^{\prime}} \int_{0}^{\mu(t)}\left[|f-V u|^{*}(r)\right]^{2} d r\left[-\left.\frac{d}{d t} \int_{\{|\nabla u|>t\}}|\nabla| \nabla u(x)\right|^{2} d x\right]^{1 / 2} \\
& \quad+C t^{2}\left[-\mu^{\prime}(t)\right]^{1 / 2}(\operatorname{tr} \mathcal{B})^{* *}\left([\mu(t)]^{1 / n^{\prime}}\right)\left[-\left.\frac{d}{d t} \int_{\{|\nabla u|>t\}}|\nabla| \nabla u(x)\right|^{2} d x\right]^{1 / 2},
\end{aligned}
$$

which, together with (2.6) again, further implies that, for almost every $t \in\left(t_{u}, \infty\right)$,

$$
\begin{aligned}
t \leq & t\left[-\mu^{\prime}(t)\right]^{1 / 2}[\mu(t)]^{-1 / n^{\prime}}\left[-\frac{d}{d t} \int_{\{|\nabla u|>t\}}|f(x)-V(x) u(x)|^{2} d x\right]^{1 / 2} \\
& -\mu^{\prime}(t)[\mu(t)]^{-2 / n^{\prime}} \int_{0}^{\mu(t)}\left[|f-V u|^{*}(r)\right]^{2} d r
\end{aligned}
$$




$$
-C t^{2} \mu^{\prime}(t)[\mu(t)]^{-1 / n^{\prime}}(\operatorname{tr} \mathcal{B})^{* *}\left([\mu(t)]^{1 / n^{\prime}}\right) .
$$

Furthermore, from the fact that $|\nabla u| \in W^{1,2}(\Omega)$, we deduce that $|\nabla u|^{*}$ is continuous and $|\nabla u|^{*}(\mu(t))=t$ for all $t \in(0, \infty)$ (see, for example, [4, Lemma 6.6]). Define the function $\phi_{V}:(0,|\Omega|) \rightarrow[0, \infty)$ as, for $s \in(0,|\Omega|)$,

$$
\phi_{V}(s):=\left\{\frac{d}{d s} \int_{\left\{|\nabla u|>|\nabla u|^{*}(s)\right\}}|f(x)-V(x) u(x)|^{2} d x\right\}^{1 / 2} .
$$

Then, for almost every $t \in(0, \infty)$,

$$
\left\{-\frac{d}{d t} \int_{\{|\nabla u|>t\}}|f(x)-V(x) u(x)|^{2} d x\right\}^{1 / 2}=\left[-\mu^{\prime}(t)\right]^{1 / 2} \phi_{V}(\mu(t)),
$$

which, combined with (3.12), further implies that, for almost $t \in\left(t_{u}, \infty\right)$,

$$
\begin{aligned}
t \leq & -t \mu^{\prime}(t)[\mu(t)]^{-1 / n^{\prime}} \phi_{V}(\mu(t))-\mu^{\prime}(t)[\mu(t)]^{-2 / n^{\prime}} \int_{0}^{\mu(t)}\left[|f-V u|^{*}(r)\right]^{2} d r \\
& -C t^{2} \mu^{\prime}(t)[\mu(t)]^{-1 / n^{\prime}}(\operatorname{tr} \mathcal{B})^{* *}\left([\mu(t)]^{1 / n^{\prime}}\right) .
\end{aligned}
$$

Let $t_{u} \leq t_{0}<T<\|\nabla u\|_{L^{\infty}(\Omega)}$. Then by (3.14), we conclude that

$$
\begin{aligned}
T^{2} \leq & t_{0}^{2}+2 \int_{t_{0}}^{T} t\left(-\mu^{\prime}(t)\right)[\mu(t)]^{-1 / n^{\prime}} \phi_{V}(\mu(t)) d t \\
& +2 \int_{t_{0}}^{T}\left(-\mu^{\prime}(t)\right)[\mu(t)]^{-2 / n^{\prime}} \int_{0}^{\mu(t)}\left[|f-V u|^{*}(r)\right]^{2} d r d t \\
& +2 C \int_{t_{0}}^{T} t^{2}\left(-\mu^{\prime}(t)\right)[\mu(t)]^{-1 / n^{\prime}}(\operatorname{tr} \mathcal{B})^{* *}\left([\mu(t)]^{1 / n^{\prime}}\right) d t \\
\leq & t_{0}^{2}+2 T \int_{0}^{\mu\left(t_{0}\right)} s^{-1 / n^{\prime}} \phi_{V}(s) d s+2 \int_{0}^{\mu\left(t_{0}\right)} s^{-2 / n^{\prime}} \int_{0}^{s}\left[|f-V u|^{*}(r)\right]^{2} d r d s \\
& +2 C T^{2} \int_{0}^{\mu\left(t_{0}\right)^{1 / n^{\prime}}}(\operatorname{tr} \mathcal{B})^{* *}(s) s^{(2-n) /(n-1)} d s
\end{aligned}
$$

Let $G:[0, \infty) \rightarrow[0, \infty)$ is defined by, for $s \in[0, \infty)$,

$$
G(s):=C \int_{0}^{s^{1 / n^{\prime}}}(\operatorname{tr} \mathcal{B})^{* *}(r) r^{(2-n) /(n-1)} d r .
$$

Choose $s_{0}:=\min \left\{\alpha|\Omega|, G^{-1}(1 / 4 C)\right\}$ and $t_{0}:=|\nabla u|^{*}\left(s_{0}\right)$. Then $t_{0} \geq t_{u}$. From Lemma 2.6 , it follows that $\mu\left(t_{0}\right)=\mu\left(|\nabla u|^{*}\left(s_{0}\right)\right) \leq s_{0} \leq G^{-1}(1 / 4 C)$, and hence

$$
C \int_{0}^{\mu\left(t_{0}\right)^{1 / n^{\prime}}}(\operatorname{tr} \mathcal{B})^{* *}(s) s^{(2-n) /(n-1)} d s \leq \frac{1}{4},
$$

which, together with (3.15), implies that

$$
T^{2} \lesssim t_{0}^{2}+T \int_{0}^{|\Omega|} s^{-1 / n^{\prime}} \phi_{V}(s) d s+\int_{0}^{|\Omega|} s^{-2 / n^{\prime}} \int_{0}^{s}\left[|f-V u|^{*}(r)\right]^{2} d r d s .
$$


Moreover, by Lemma 2.2, we find that, for any $s \in(0,|\Omega|)$,

$$
\int_{0}^{s}\left[\phi_{V}^{*}(r)\right]^{2} d r \leq \int_{0}^{s}\left[|f-V u|^{*}(r)\right]^{2} d r
$$

which, combined with Lemma 2.3, implies that

$$
\int_{0}^{|\Omega|} s^{-1 / n^{\prime}} \phi_{V}(s) d s \lesssim\|f-V u\|_{L^{n, 1}(\Omega)} .
$$

Furthermore, from Lemma 2.4, we deduce that

$$
\int_{0}^{|\Omega|} s^{-2 / n^{\prime}} \int_{0}^{s}\left[|f-V u|^{*}(r)\right]^{2} d r d s \lesssim\|f-V u\|_{L^{n, 1}(\Omega)}^{2},
$$

which, together with (3.16) and (3.17), further implies that

$$
T^{2} \lesssim t_{0}^{2}+T\|f-V u\|_{L^{n, 1}(\Omega)}+\|f-V u\|_{L^{n, 1}(\Omega)}^{2} .
$$

By this and Hölder's inequality, we conclude that

$$
T \lesssim t_{0}+\|f-V u\|_{L^{n, 1}(\Omega)} .
$$

Moreover, from the equality

$$
\int_{\Omega}|\nabla u(x)|^{2} d x+\int_{\Omega} V(x)|u(x)|^{2} d x=\int_{\Omega} f(x) u(x) d x,
$$

Hölder's inequality (1.13) and Sobolev's inequality, it follows that

$$
\begin{aligned}
\int_{\Omega}|\nabla u(x)|^{2} d x & \lesssim\|f-V u\|_{L^{n, 1}(\Omega)}\|u\|_{L^{n^{\prime}, \infty}(\Omega)} \lesssim\|f-V u\|_{L^{n, 1}(\Omega)}\|u\|_{L^{n^{\prime}}(\Omega)} \\
& \lesssim\|f-V u\|_{L^{n, 1}(\Omega)}\|\nabla u\|_{L^{1}(\Omega)},
\end{aligned}
$$

which implies that $\|\nabla u\|_{L^{2}(\Omega)} \lesssim\|f-V u\|_{L^{n, 1}(\Omega)}$. By this, we find that

$$
\|f-V u\|_{L^{n, 1}(\Omega)}^{2} \gtrsim\|\nabla u\|_{L^{2}(\Omega)}^{2} \gtrsim \int_{\left\{|\nabla u| \geq t_{0}\right\}}|\nabla u(x)|^{2} d x \gtrsim t_{0}^{2}|\Omega|,
$$

which, combined with (3.18), further implies that $T \lesssim\|f-V u\|_{L^{n, 1}(\Omega)}$. Letting $T \rightarrow$ $\|\nabla u\|_{L^{\infty}(\Omega)}$, we see that (3.5) holds true.

Step 4. From Step 3, we deduce that

$$
\|\nabla u\|_{L^{\infty}(\Omega)}<\infty
$$

which further implies that $u \in W_{0}^{1, \infty}(\Omega) \cap W^{2,2}(\Omega)$. Then there exists a sequence $\left\{u_{k}\right\}_{k \in \mathbb{N}} \subset C^{\infty}(\Omega) \cap C^{2}(\bar{\Omega})$ satisfying (3.1), (3.2) and that, for any $k \in \mathbb{N}, u_{k}=0$ on $\partial \Omega$, and

$$
\left\|\nabla u_{k}\right\|_{L^{\infty}(\Omega)} \rightarrow\|\nabla u\|_{L^{\infty}(\Omega)} \text { as } k \rightarrow \infty
$$


Notice that (2.7) holds true for $u_{k}$. Then an argument similar to that in Step 2 yields that, for almost every $t \in(0, \infty)$,

$$
\begin{aligned}
& t \int_{\{|\nabla u|=t\}}|\nabla| \nabla u(x)|| d \mathcal{H}^{n-1} \\
& \quad \leq t \int_{\{|\nabla u|=t\}}|f(x)-V(x) u(x)| d \mathcal{H}^{n-1}+\int_{\{|\nabla u|>t\}}|f(x)-V(x) u(x)|^{2} d x \\
& \quad+\|\nabla u\|_{L^{\infty}(\Omega)}^{2} \int_{\partial \Omega \cap \partial\{|\nabla u|>t\}}|\operatorname{tr} \mathcal{B}(x)| d \mathcal{H}^{n-1} .
\end{aligned}
$$

By replacing (3.4) with (3.19) and repeating the proof of (3.5), we conclude that there exists a positive constant $C$, depending on $n,\|\operatorname{tr} \mathcal{B}\|_{L^{n-1,1}(\partial \Omega)},|\Omega|$ and the constant in (2.2), such that

$$
\|\nabla u\|_{L^{\infty}(\Omega)} \leq C\|f-V u\|_{L^{n, 1}(\Omega)} .
$$

Step 5. In this step, we prove that

$$
\|\nabla u\|_{L^{\infty}(\Omega)} \leq C\|f\|_{L^{n, 1}(\Omega)},
$$

where $C$ is a positive constant depending on $n,\|\operatorname{tr} \mathcal{B}\|_{L^{n-1,1}(\partial \Omega)},|\Omega|$ and the constant in $(2.2)$.

Denote by $G_{\Omega}$ and $\Gamma$ the Green function of the operator $L_{\Omega}:=-\Delta+V$, with the Dirichlet boundary condition, on $\Omega$ and the fundamental solution of $L_{\mathbb{R}^{n}}:=-\Delta+V$ on $\mathbb{R}^{n}$. Let $p \in\left(1, q_{+}\right)$, with $q_{+}$as in $(1.1)$, and $g \in L^{p}(\Omega)$. Denote by $\widetilde{g}$ the zero extension of $g$ on $\mathbb{R}^{n}$. Let $\widetilde{g}^{+}:=\max \{g, 0\}$ and $\widetilde{g}^{-}:=-\min \{g, 0\}$. Then $\widetilde{g}=\widetilde{g}^{+}-\widetilde{g}^{-}$and $\left\|\widetilde{g}^{+}\right\|_{L^{p}\left(\mathbb{R}^{n}\right)}+\left\|\widetilde{g}^{-}\right\|_{L^{p}\left(\mathbb{R}^{n}\right)} \leq 2\|\widetilde{g}\|_{L^{p}\left(\mathbb{R}^{n}\right)}$. From [24, Theorem 3.1], we deduce that

$$
\left\|V L_{\mathbb{R}^{n}}^{-1}(\widetilde{g})\right\|_{L^{p}\left(\mathbb{R}^{n}\right)} \lesssim\|\widetilde{g}\|_{L^{p}\left(\mathbb{R}^{n}\right)}
$$

By this, $V \geq 0$ and the fact that, for all $x, y \in \Omega, 0 \leq G_{\Omega}(x, y) \leq \Gamma(x, y)$, which is a simple corollary of the classical maximum principle, we further conclude that

$$
\begin{aligned}
\left\|V L_{\Omega}^{-1}(g)\right\|_{L^{p}(\Omega)} & \leq\left\|V L_{\Omega}^{-1}\left(g^{+}\right)\right\|_{L^{p}(\Omega)}+\left\|V L_{\Omega}^{-1}\left(g^{-}\right)\right\|_{L^{p}(\Omega)} \\
& \leq\left\|V L_{\mathbb{R}^{n}}^{-1}\left(\widetilde{g}^{+}\right)\right\|_{L^{p}\left(\mathbb{R}^{n}\right)}+\left\|V L_{\mathbb{R}^{n}}^{-1}\left(\widetilde{g}^{-}\right)\right\|_{L^{p}\left(\mathbb{R}^{n}\right)} \\
& \lesssim\left\|\widetilde{g}^{+}\right\|_{L^{p}\left(\mathbb{R}^{n}\right)}+\left\|\widetilde{g}^{-}\right\|_{L^{p}\left(\mathbb{R}^{n}\right)} \lesssim\|\widetilde{g}\|_{L^{p}\left(\mathbb{R}^{n}\right)} \sim\|g\|_{L^{p}(\Omega)}
\end{aligned}
$$

Thus, the operator $V L_{\Omega}^{-1}$ is bounded on $L^{p}(\Omega)$ with $p \in\left(1, q_{+}\right)$.

From $V \in R H_{n}\left(\mathbb{R}^{n}\right)$ and the self-improvement property of $R H_{n}\left(\mathbb{R}^{n}\right)$, it follows that $q_{+}>n$. Take $q_{1}, q_{2} \in\left(1, q_{+}\right)$such that $q_{1}<n<q_{2}$. For any measurable set $E \subset \Omega$, by (3.22), we find that $\left\|V L_{\Omega}^{-1}\left(\chi_{E}\right)\right\|_{L^{q_{i}(\Omega)}} \lesssim\left\|\chi_{E}\right\|_{L^{q_{i}(\Omega)}}$, with $i \in\{1,2\}$, which further implies that

$$
\left\|V L_{\Omega}^{-1}\left(\chi_{E}\right)\right\|_{L^{q_{i}, \infty}(\Omega)} \lesssim|E|^{1 / q_{i}},
$$

where $i \in\{1,2\}$. From this and Lemma 3.1, we deduce that, for any $q \in\left(q_{1}, q_{2}\right)$ and $r \in(0, \infty)$, the operator $V L_{\Omega}^{-1}$ is bounded on the space $L^{q, r}(\Omega)$. In particular, $V L_{\Omega}^{-1}$ is bounded on $L^{n, 1}(\Omega)$, which, combined with $u=L_{\Omega}^{-1} f$, implies that

$$
\|V u\|_{L^{n, 1}(\Omega)} \lesssim\|f\|_{L^{n, 1}(\Omega)} .
$$


By this and (3.20), we conclude that (3.21) holds true.

Step 6. In this step, we remove the assumption $\partial \Omega \in C^{\infty}$ for $\Omega$.

From the fact that, for any open set $U \in \mathbb{R}^{n-1}$, the space $C^{\infty}(U) \cap W^{2} L^{n-1,1}(U)$ is dense in $W^{2} L^{n-1,1}(U)$, it follows that there exists a sequence $\left\{\Omega_{m}\right\}_{m \in \mathbb{N}}$ of bounded domains such that, for all $m \in \mathbb{N}, \Omega \subset \Omega_{m}$ and $\partial \Omega_{m} \in C^{\infty},\left|\Omega_{m} \backslash \Omega\right| \rightarrow 0$ and $\Omega_{m} \rightarrow \Omega$ with respect to the Hausdorff distance, as $m \rightarrow \infty$, and $\left\|\operatorname{tr} \mathcal{B}_{m}\right\|_{L^{n-1,1}(\partial \Omega)} \leq C$ for some positive constant $C$ depending on $\Omega$, where $\operatorname{tr} \mathcal{B}_{m}$ denotes the trace of the second fundamental form on $\partial \Omega_{m}$. We remark that the sequence $\left\{\Omega_{m}\right\}_{m \in \mathbb{N}}$ could be chosen satisfying that the constant in (2.2), with $\Omega$ replaced with $\Omega_{m}$, are bounded, up to a multiplicative constant independent of $m$, by the corresponding constant for $\Omega$. In fact, as the argument in [6, p. 170], we know that, for any domain $U \subset \mathbb{R}^{n-1}$, the embedding $W^{2} L^{n-1,1}(U) \rightarrow W^{1, \infty}(U)$ further implies that the convergence of the Lipschitz constants of the functions whose graphs locally agree with $\partial \Omega_{m}$ to the Lipschitz constant of the function whose graph coincides with $\partial \Omega$.

For any $m \in \mathbb{N}$, let

$$
f_{m}:= \begin{cases}f & \text { in } \Omega \\ 0 & \text { in } \Omega_{m} \backslash \Omega .\end{cases}
$$

Denote by $u_{m}$ the weak solution of the problem (1.2) with $\Omega$ and $f$ replaced with $\Omega_{m}$ and $f_{m}$, respectively. Then for any $m \in \mathbb{N}, u_{m} \in W_{\text {loc }}^{2,2}(\Omega)$ and from Remark 1.1(ii) and (3.1), we deduce that, for every open set $\Omega_{0}$ with $\bar{\Omega}_{0} \subset \Omega$, there exists a positive constant $C$, depending on $\Omega, f$ and $V$, such that, for all $m \in \mathbb{N}$,

$$
\left\|u_{m}\right\|_{W^{2,2}\left(\Omega_{0}\right)} \leq C .
$$

Moreover, by (3.21) with $\Omega$ replaced with $\Omega_{m}$ and $u$ replaced with $u_{m}$, we know that there exists a positive constant $C$ such that, for all $m \in \mathbb{N}$,

$$
\left\|\nabla u_{m}\right\|_{L^{\infty}(\Omega)} \leq C .
$$

Let $s \in[1,2 n /(n-2))$. If $\partial \Omega_{0}$ is smooth, then the embedding $W^{2,2}\left(\Omega_{0}\right) \rightarrow W^{1, s}\left(\Omega_{0}\right)$ is compact. From (3.24) and (3.25), we deduce that there exists $u \in W^{1, \infty}(\Omega)$ and a subsequence of $\left\{u_{m}\right\}_{m \in \mathbb{N}}$, still denote by $\left\{u_{m}\right\}_{m \in \mathbb{N}}$, such that

$$
u_{m} \rightarrow u \text { in } W_{\text {loc }}^{1, s}(\Omega)
$$

and

$$
\nabla u_{m} \rightarrow \nabla u \text { almost everywhere in } \Omega \text {. }
$$

By $u_{m}=0$ on $\partial \Omega_{m}, \Omega_{m} \rightarrow \Omega$ in the Hausdorff distance and (3.25), we know that $u=0$ on $\partial \Omega$. Thus, $u \in W_{0}^{1,2}(\Omega)$. From the facts that $u_{m}$ is the weak solution of the Dirichlet problem (1.2) and $\Omega \subset \Omega_{m}$ for any $m \in \mathbb{N}$, it follows that, for any $\psi \in C_{c}^{\infty}(\Omega)$,

$$
\int_{\Omega_{m}} \nabla u_{m}(x) \cdot \nabla \psi(x) d x+\int_{\Omega_{m}} V(x) u_{m}(x) \psi(x) d x=\int_{\Omega_{m}} f(x) \psi(x) d x .
$$

Letting $m \rightarrow \infty$ in (3.27), via (3.25), (3.26) and the dominated convergence theorem, we conclude that, for any $\psi \in C_{c}^{\infty}(\Omega)$,

$$
\int_{\Omega} \nabla u(x) \cdot \nabla \psi(x) d x+\int_{\Omega} V(x) u(x) \psi(x) d x=\int_{\Omega} f(x) \psi(x) d x
$$


which, together with the fact that $C_{c}^{\infty}(\Omega)$ is dense in $W_{0}^{1,2}(\Omega)$, implies that $u$ is the weak solution of the problem (1.2). Moreover, it follows, from (3.26) and the fact for any $m \in \mathbb{N}, u_{m}$ satisfies $(3.21)$, that $\|\nabla u\|_{L^{\infty}(\Omega)} \lesssim\|f\|_{L^{n, 1}(\Omega)}$, which finishes the proof of Theorem 1.2.

To prove Theorem 1.3, we need the following Lemma 3.2, whose proof is similar to that of [23, Lemma 6.4], the details being omitted here.

Lemma 3.2. Let $n \geq 2$ and $\Omega$ be a bounded semi-convex domain in $\mathbb{R}^{n}$. Then there exists a sequence of bounded semi-convex domain $\left\{\Omega_{j}\right\}_{j \in \mathbb{N}}$ such that $\bar{\Omega} \subset \Omega_{j}$ and $\partial \Omega_{j} \in C^{\infty}$ for all $j \in \mathbb{N},\left|\Omega_{j} \backslash \Omega\right| \rightarrow 0$ and $\Omega_{j} \rightarrow \Omega$ with respect to the Hausdorff distance, as $j \rightarrow \infty$. Moreover, for any $j \in \mathbb{N}, \Omega_{j}$ has the uniform Lipschitz character and the uniform ball constant with $\Omega$.

Now we prove Theorem 1.3 by using Lemma 3.2 .

Proof of Theorem 1.3. The proof of Theorem 1.3 is similar that of Theorem 1.2. More precisely, we repeat the proof of Theorem 1.2 by replacing (2.7) and (2.8) with (2.9) and (2.10), respectively. Moreover, from Lemma 3.2, we deduce that there exists a sequence $\left\{\Omega_{m}\right\}_{m \in \mathbb{N}}$ of bounded, semi-convex and smooth domains such that $\left|\Omega_{m} \backslash \Omega\right| \rightarrow 0$ and $\Omega_{m} \rightarrow \Omega$ with respect to the Hausdorff distance as $m \rightarrow \infty$, which is employed in Step 6.

To prove Theorem 1.4, we need the notion of Neumann functions and some associated estimates. Let $n \geq 3$ and $\Omega \subset \mathbb{R}^{n}$ be a bounded Lipschitz domain. By [25, (1.20)], we know that there exists a function $N(\cdot, \cdot)$ on $\Omega \times \Omega$ such that, for any $x, y \in \Omega$,

$$
\begin{cases}-\Delta_{x} N(x, y)+V(x) N(x, y)=\delta_{y}(x), & \text { in } \Omega \\ \frac{\partial}{\partial \nu} N(\cdot, y)=0, & \text { on } \partial \Omega .\end{cases}
$$

The function $N$ is called the Neumann function. Moreover, for the potential $V \in R H_{q}\left(\mathbb{R}^{n}\right)$, with $q \in[n / 2, \infty)$, the auxiliary function $m(x, V)$ associated with $V$ is defined by, for all $x \in \mathbb{R}^{n}$,

$$
[m(x, V)]^{-1}:=\sup \left\{r \in(0, \infty): \frac{r^{2}}{|B(x, r)|} \int_{B(x, r)} V(y) d y \leq 1\right\},
$$

which was introduced by Shen [24]. For the Neumann function $N$, we have the following estimate, which was established by Shen [25, Lemma 1.21].

Lemma 3.3. Let $n \geq 3$ and $\Omega \subset \mathbb{R}^{n}$ be a bounded Lipschitz domain. Then for any $k \in \mathbb{N}$, there exists a positive constant $C$, depending on $n$ and $k$, such that, for any $x, y \in \Omega$,

$$
|N(x, y)| \leq \frac{C}{[1+|x-y| m(x, V)]^{k}} \frac{1}{|x-y|^{n-2}} .
$$

Proof of Theorem 1.4. The proof of Theorem 1.4 is similar to that of Theorem 1.2 for the Dirichlet case. Here we point out some slight variants.

Step 1. Assume that $\partial \Omega \in C^{\infty}$ and $u$ is the weak solution of the Neumann problem (1.4). Then from $f \in L^{n, 1}(\Omega) \subset L^{2}(\Omega)$ and Remark 1.1(iii), we deduce that $u \in W^{2,2}(\Omega)$. 
Moreover, by $[6,(6.95)]$, we know that there exists a sequence $\left\{u_{k}\right\}_{k \in \mathbb{N}} \subset C^{\infty}(\Omega) \cap C^{2}(\bar{\Omega})$ such that, for any $k \in \mathbb{N}, \frac{\partial u_{k}}{\partial \nu}=0$ on $\partial \Omega$,

$$
u_{k} \rightarrow u \text { in } W^{2,2}(\Omega) \text { and } \nabla u_{k} \rightarrow \nabla u \text { almost everywhere in } \Omega .
$$

Via replacing (3.1) with (3.29), then an argument, similar to that in Step 1 in the proof of Theorem 1.2, yields that the sequence $\left\{u_{k}\right\}_{k \in \mathbb{N}}$ satisfies (3.2).

Step 2. By replacing (2.8) with (2.24) and repeating the proof of Step 2 in the proof of Theorem 1.2, we conclude that, for almost every $t \in\left(t_{u}, \infty\right)$,

$$
\begin{aligned}
& t \int_{\{|\nabla u|=t\}}|\nabla| \nabla u(x)|| d \mathcal{H}^{n-1} \\
& \quad \leq t \int_{\{|\nabla u|=t\}}|f(x)-V(x) u(x)| d \mathcal{H}^{n-1}+\int_{\{|\nabla u|>t\}}|f(x)-V(x) u(x)|^{2} d x \\
& \quad+2 t^{2} \int_{\partial \Omega \cap \partial\{|\nabla u|>t\}}|\widetilde{\mathcal{B}}(x)| d \mathcal{H}^{n-1} .
\end{aligned}
$$

Step 3. Via replacing $|\operatorname{tr} \mathcal{B}(x)|$ and (3.4) with $|\widetilde{\mathcal{B}}(x)|$ and (3.30) respectively, then an argument similar to that in Step 3 in the proof of Theorem 1.2, yields that, for any given $r \in(n-1, \infty)$

$$
\|\nabla u\|_{L^{\infty}(\Omega)} \lesssim\|f-V u\|_{L^{n, 1}(\Omega)}
$$

where the implicit constant depends on $n, r,\||\widetilde{\mathcal{B}}|\|_{L^{r}(\partial \Omega)},|\Omega|$ and the constant in (2.2).

Step 4. Replacing (2.7) with (2.23) and repeating the proof of Step 4 in the proof of Theorem 1.2, we find that (3.31) holds true with the implicit constant depending on $n$, $\||\widetilde{\mathcal{B}}|\|_{L^{n-1,1}(\partial \Omega)},|\Omega|$ and the constant in $(2.2)$.

Step 5. In this step, we prove that

$$
\|\nabla u\|_{L^{\infty}(\Omega)} \lesssim\|f\|_{L^{n, 1}(\Omega)}
$$

where the implicit constant depends on $n,\left\|\left|\widetilde{\mathcal{B}} \|_{L^{n-1,1}(\partial \Omega)},\right| \Omega \mid\right.$ and the constant in (2.2). Let $L_{\Omega}:=-\Delta+V$ with the Neumann boundary condition and $N$ be the Neumann function associated with $L_{\Omega}$. Assume that $p \in\left[1, q_{+}\right)$and $g \in L^{p}(\Omega)$. We claim that

$$
\left\|V L_{\Omega}^{-1}(g)\right\|_{L^{p}(\Omega)} \lesssim\|g\|_{L^{p}(\Omega)}
$$

Once (3.33) holds true, then by (3.33), Lemma 3.1 and $u=L_{\Omega}^{-1} f$, similar to the proof of (3.23), we conclude that $\|V u\|_{L^{n, 1}(\Omega)} \lesssim\|f\|_{L^{n, 1}(\Omega)}$, which, combined with (3.31), further implies that (3.32) holds true.

Now we give out the proof of (3.33). For any $x \in \Omega$, let $w(x):=\int_{\Omega} N(x, y) g(y) d y$. Then the inequality (3.33) is equivalent to

$$
\|V w\|_{L^{p}(\Omega)} \lesssim\|g\|_{L^{p}(\Omega)} .
$$

For any $x, y \in \Omega$, let

$$
\widetilde{N}(x, y):=\left\{\begin{array}{ll}
N(x, y), & x, y \in \Omega, \\
0, & \text { else },
\end{array} \widetilde{g}(x):= \begin{cases}g(x), & x \in \Omega, \\
0, & x \in \mathbb{R}^{n} \backslash \Omega,\end{cases}\right.
$$


and $\widetilde{w}(x):=\int_{\mathbb{R}^{n}} \widetilde{N}(x, y) \widetilde{g}(y) d y$. Then $\widetilde{g} \in L^{p}\left(\mathbb{R}^{n}\right)$ and from Lemma 3.3, it follows that, for any $k \in \mathbb{N}$ and $x, y \in \mathbb{R}^{n}$,

$$
|\widetilde{N}(x, y)| \lesssim \frac{1}{[1+|x-y| m(x, V)]^{k}} \frac{1}{|x-y|^{n-2}} .
$$

Via using these estimates and repeating the proof of [24, Theorem 3.1], we obtain that $\|V \widetilde{w}\|_{L^{p}\left(\mathbb{R}^{n}\right)} \lesssim\|\widetilde{g}\|_{L^{p}\left(\mathbb{R}^{n}\right)}$, which implies that (3.34) holds true.

Step 6. Let $\left\{\Omega_{m}\right\}_{m \in \mathbb{N}}$ be as in Step 6 in the proof of Theorem 1.2. Then we obtain a corresponding sequence $\left\{u_{m}\right\}_{m \in \mathbb{N}}$ of solution to the Neumann problems in $\left\{\Omega_{m}\right\}_{m \in \mathbb{N}}$, which satisfy $(3.24),(3.25),(3.26)$ and $(3.27)$ for all $\psi \in \operatorname{Lip}\left(\mathbb{R}^{n}\right)$. By (3.27) and letting $m \rightarrow \infty$, we see that (3.28) holds true for any $\psi \in \operatorname{Lip}\left(\mathbb{R}^{n}\right)$, which, together with the facts that $\Omega$ is a bounded Lipschitz domain and the space of the restrictions to $\Omega$ of the functions from $\operatorname{Lip}\left(\mathbb{R}^{n}\right)$ is dense in $W^{1,2}(\Omega)$, implies that $u$ is the weak solution of the Neumann problem (1.4). Moreover, it follows, from (3.26) and the fact for any $m \in \mathbb{N}$, $u_{m}$ satisfies (3.32), that $\|\nabla u\|_{L^{\infty}(\Omega)} \lesssim\|f\|_{L^{n, 1}(\Omega)}$, which finishes the proof of Theorem 1.4 .

Finally we prove Theorem 1.5.

Proof of Theorem 1.5. The proof of Theorem 1.5 is similar that of Theorem 1.4. More precisely, by using (2.25), (2.26) and Lemma 3.2, and repeating the proof of Theorem 1.4, we finish the proof of Theorem 1.5, the details being omitted here.

Acknowledgement. The author would like to thank Professor Jun Geng for helpful discussions on this topic.

\section{References}

[1] A. Alvino, A. Cianchi, V. G. Maz'ya and A. Mercaldo, Well-posed elliptic Neumann problems involving irregular data and domains, Ann. Inst. H. Poincaré Anal. Non Linéaire 27 (2010), 1017-1054.

[2] J. Brothers and W. Ziemer, Minimal rearrangements of Sobolev functions, J. Reine Angew. Math. 384 (1988), 153-179.

[3] A. Cianchi, Maximizing the $L^{\infty}$-norm of the gradient of solutions to the Poisson equation, J. Geom. Anal. 2 (1992), 499-515.

[4] A. Cianchi, D. Edmunds and P. Gurka, On weighted Poincaré inequalities, Math. Nachr. 180 (1996), 15-41.

[5] A. Cianchi and V. G. Maz'ya, Global gradient estimates in elliptic problems under minimal data and domain regularity, Commun. Pure Appl. Anal. 14 (2015), 285-311.

[6] A. Cianchi and V. G. Maz'ya, Global boundedness of the gradient for a class of nonlinear elliptic systems, Arch. Ration. Mech. Anal. 212 (2014), 129-177.

[7] A. Cianchi and V. G. Maz'ya, Gradient regularity via rearrangements for $p$-Laplacian type elliptic boundary value problems, J. Eur. Math. Soc. 16 (2014), 571-595.

[8] A. Cianchi and V. G. Maz'ya, Global Lipschitz regularity for a class of quasilinear elliptic equations, Comm. Partial Differential Equations 36 (2011), 100-133. 
[9] A. Cianchi and L. Pick, Sobolev embeddings into BMO, VMO, and $L^{\infty}$, Ark. Mat. 36 (1998), 317-340.

[10] E. Di Benedetto, $C^{1+\alpha}$ local regularity of weak solutions of degenerate elliptic equations, Nonlinear Anal. 7 (1983), 827-850.

[11] H. Eggleston, Convexity, Cambridge Tracts in Mathematics and Mathematical Physics, No. 47, Cambridge University Press, New York, 1958.

[12] F. Gehring, The $L^{p}$-integrability of the partial derivatives of a quasiconformal mapping, Acta Math. 130 (1973), 265-277.

[13] D. Gilbarg and N. S. Trudinger, Elliptic Partial Differential Equations of Second Order, Reprint of the 1998 edition, Springer-Verlag, Berlin, 2001.

[14] L. Grafakos, Classical Fourier Analysis, Third edition, Graduate Texts in Mathematics 249, Springer, New York, 2014.

[15] P. Grisvard, Elliptic Problems in Nonsmooth Domains, Monographs and Studies in Mathematics, 24, Boston, MA, 1985.

[16] V. G. Maz'ya, Sobolev Spaces with Applications to Elliptic Partial Differential Equations, Springer, Heidelberg, 2011.

[17] V. G. Maz'ya, On the boundedness of first derivatives for solutions to the NeumannLaplace problem in a convex domain, J. Math. Sci. (N. Y.) 159 (2009), 104-112.

[18] V. G. Maz'ya, Boundedness of the gradient of a solution to the Neumann-Laplace problem in a convex domain, C. R. Math. Acad. Sci. Paris 347 (2009), 517-520.

[19] V. G. Mazya, On weak solutions of the Dirichlet and Neumann problems, Trans. Moscow Math. Soc. 20 (1969), 135-172.

[20] D. Mitrea, I. Mitrea, M. Mitrea and L. Yan, Coercive energy estimates for differential forms in semi-convex domains, Commun. Pure Appl. Anal. 9 (2010), 987-1010.

[21] D. Mitrea, I. Mitrea, M. Mitrea and L. Yan, On the geometry of domains satisfying uniform ball conditions, preprint (2009).

[22] D. Mitrea, M. Mitrea and L. Yan, Boundary value problems for the Laplacian in convex and semiconvex domains, J. Funct. Anal. 258 (2010), 2507-2585.

[23] M. Mitrea, Dirichlet integrals and Gaffney-Friedrichs inequalities in convex domains, Forum Math. 13 (2001), 531-567.

[24] Z. Shen, $L^{p}$ estimates for Schrödinger operators with certain potentials, Ann. Inst. Fourier (Grenoble) 45 (1995), 513-546.

[25] Z. Shen, On the Neumann problem for Schrödinger operators in Lipschitz domains, Indiana Univ. Math. J. 43 (1994), 143-176.

[26] G. Talenti, Elliptic equations and rearrangements, Ann. Scuola Norm. Sup. Pisa Cl. Sci. (4) 3 (1976), 697-718.

[27] G. Talenti, Nonlinear elliptic equations, rearrangements of functions and Orlicz spaces, Ann. Mat. Pura Appl. (4) 120 (1979), 160-184.

Sibei Yang

School of Mathematics and Statistics, Gansu Key Laboratory of Applied Mathematics and Complex Systems, Lanzhou University, Lanzhou, Gansu 730000, People's Republic of China

E-mail: yangsb@lzu.edu.cn 